\title{
The Influence of Personality and Demographic Characteristics on Aggressive Driving Behaviors in Eastern Chinese Drivers
}

\author{
Xiao-kun Liu' \\ Shan-lin Chen' \\ Dan-ling Huang' \\ Zi-shang Jiang' \\ Yu-ting Jiang (D) \\ Li-juan Liang' \\ Lu-lu Qin $\mathbb{1 D}^{2}$
}

'The First Affiliated Hospital of Hainan Medical University, Haikou, People's Republic of China; ${ }^{2}$ Department of Social Medicine and Health Management, School of Medicine, Hunan Normal University, Changsha, People's Republic of China
Correspondence: Xiao-kun Liu The First Affiliated Hospital of Hainan Medical University, 3 Xueyuan Road, Haikou, 57I 199, Hainan, People's

Republic of China

Email lacyfairy@qq.com
Purpose: Road safety research is important due to the large number of road traffic fatalities globally. This study investigated the influences of age, driving experience and other covariates on aggressive driving behavior.

Methods: A cross-sectional survey was conducted in Yixing City, Wuxi City, Jiangsu Province, China. Regression analysis was applied to explore the influences of age and driving experience and their interactions with other covariates on aggressive driving behavior. Two analyses methodologies were used to assess the simple effect of the interactions. Firstly, the Jamovi automatic analysis classification program was used to calculate the simple slope test. Second, the SPSS macro program was also used to calculate the simple slope test also.

Results: A total of 570 drivers (247 males, 282 females) participated in the survey. A negative correlation was found between age and aggressive driving behaviors, and a positive correlation was found between neuroticism and aggressive driving behaviors in the multiple regression analysis. Significant associations were also found between age, driving experience, and depression, as well as age, driving experience, and neuroticism. Simple slope tests showed that depressive symptoms could increase aggressive behaviors in the elderly and experienced drivers. When experiencing neuroticism, individuals with higher driving experience were more aggressive in driving than shorter experienced drivers.

Conclusion: Age and neuroticism influenced aggressive driving behaviors. Veteran drivers could be aggressive drivers when experiencing depressive symptoms or neuroticism. Mobile intervention could be sent to the potentially risky drivers, which would be safe and broadly feasible to prevent aggressive driving behavior in the background of COVID-19.

Keywords: aggressive driving behavior, age, driving experience, depressive symptoms, neuroticism

\section{Introduction}

Road safety has been an important global public health issue, particularly over the last few decades. ${ }^{1}$ Injury resulting from traffic accidents accounted for many years lived with disability, ${ }^{2,3}$ and brought about considerable loss in productivity and economic outcomes. ${ }^{4,5}$ According to a World Health Organization report in 2019, more than 1.35 million people die on the roads annually. ${ }^{6}$ China has the largest number of road traffic fatalities in the world. ${ }^{7}$ The road fatalities in China accounted for $23 \%$ of the total global road traffic deaths in $2016,{ }^{8}$ and approximately $21 \%$ of the total global road fatalities in $2017 .{ }^{9}$ Studies have shown that aggressive driving behavior significantly contributes to road traffic 
events, including the morbidity and mortality of road traffic accident outcomes. ${ }^{10-17}$

Aggressive driving has been defined as "when individuals commit a combination of moving traffic offences to endanger other persons or property" by the National Highway Traffic Safety Administration (NHTSA). ${ }^{18,19}$ This is consistent with the definition of aggressive behavior as "any form of behavior directed toward the goal of harming or injuring another living being who is motivated to avoid such treatment". ${ }^{20}$ Studies in the field demonstrate that sociodemographic attributes are related to aggressive driving behavior. ${ }^{21-23}$

Age and aggressive driving behavior have been reported to be closely associated. Many studies have concluded that the much younger aged drivers correlated with more risky, reckless and aggressive driving behaviors, which may be associated with an increased risk of traffic accident involvement. ${ }^{24-27}$ Conversely, Chinese scholar Huihui Zhang found that older drivers undertook more aggressive driving than younger drivers. ${ }^{28} \mathrm{~A}$ Chinese high-speed railway study reported that older age was a significant predictor of accident involvement. ${ }^{29}$ Therefore, whether age and aggressive driving behavior was positively correlated or negatively has not reached consistent reporting.

Previous studies revealed that men outnumber women in causing physical injury and direct aggression. ${ }^{30-33}$ Evolutionary perspective, ${ }^{30,34}$ biosocial reformulation mechanism $^{35,36}$ and neurobiological dispositions ${ }^{37}$ may contribute to the origins of gender differences in aggressive behaviors. In cultures men may act to reassert their masculinity through aggressive behaviors. ${ }^{38}$ Dwight A. and David L. reported that violence in driving was more commonly reported in male-drivers. ${ }^{39}$ Chinese scholars Jia et al reported that males were much more likely to overtake in a driving environment. ${ }^{40}$ Additionally Feng et al reported the same phenomenon. ${ }^{41}$ Gwyther and Holland reported that women were significantly more likely to self-regulate while driving than men. ${ }^{42}$ The current studies consistently report that males are much more prone to direct aggressive behaviors.

Drivers with different driving experiences have different exposures and coping skills when negotiating conflicting traffic conditions. Lajunen and Parker (2001) found that driving experience correlated negatively with aggressive driving. ${ }^{43}$ Similarly, Chinese scholars Jia et al found that driving experience was negatively correlated with aggressive driving behavior. ${ }^{40}$ However, Chinese scholars
Li et al reported no significant correlation between aggressive driving behavior and driving experience. ${ }^{44}$ There are few reports on the relationships between driving experience and aggressive driving behaviors, so further research is required.

Up to date, most studies report that males display much more physically aggressive behaviors convergently. However, there is not a consistent understanding of the influence of age and driving experience on aggressive driving behavior. This study aims to address this research gap and explore the influences of age and driving experience, and their interactions on aggressive driving behavior. A cross-sectional survey collected the data and other confounding factors that could impact aggressive driving behavior in actual traffic environments were controlled for in the analyses including personality ${ }^{28,45,46}$ and alcohol use disorders, ${ }^{47,48}$ social supports, ${ }^{49,50}$ negative emotion (depressive and anxiety symptoms), ${ }^{11,51,52}$ and poor sleep quality. ${ }^{53,54}$ The findings of this study will contribute to understanding aggressive driving behavior and may assist in the early identification of high-risk or accident-prone drivers, thus enabling the early prevention of traffic accidents.

\section{Participants and Methods}

\section{Participants}

The incidence of traffic accidents in Eastern China (including Shanghai City, Jiangsu Province, Zhejiang Province, Anhui Province, Jiangxi Province, and Fujian Province) was higher than in other regions in China. ${ }^{55}$ The gross domestic product (GDP) of Jiangsu province was the highest among all provinces in Eastern China at the end of $2018 .{ }^{56}$ A prosperous economy was accompanied by an increase in the number of motor vehicles purchased. ${ }^{57,58}$ The per capita GDP of Wuxi City was ranked first in Jiangsu Province in 2017 and 2018..$^{59,60}$ The economic level of the Yixing area ranked in the middle of all the areas of Wuxi city. ${ }^{61}$ Therefore, participants were recruited from the Yixing area of Wuxi City.

The required sample size was estimated using the following formula: $\mathrm{n}=\mathrm{Z}^{2} \mathrm{P}(1-\mathrm{P}) / \mathrm{E}^{2}$. $\mathrm{P}$ was set at $35.7 \%$ according to previous research. ${ }^{40} \mathrm{E}$ was $5 \%$ and $\mathrm{Z}$ was 1.96. The calculated sample size was 353 . Given that $10 \%$ of the questionnaires may be invalid, the required sample size was estimated to be at least 392. In total, 570 participants completed the survey. Among these surveys, 31 
questionnaires were not correctly finished, resulting in 529 valid surveys, with a response rate of $94.56 \%$.

The inclusion criteria were: 1) adult drivers aged from 18 to 70 years, and 2) possession of a valid motor vehicle driver's license. The Application and Usages of Motor Vehicles from the Ministry of Public Security of the People's Republic of China No. 139 states that a person shall not apply for a motor vehicle driving license while experiencing mental illness conditions. Equally, those who have either taken or injected narcotic drugs during the past three years, been discharged from compulsory isolation measures for drug rehabilitation for less than three years, or have taken long-term dependent psychotropic drugs and are still addicted to them are not allowed to have a driving license. $^{62}$ Therefore having a driver's license in China means an individual is free of mental illness and drug dependence.

The exclusion criteria were: 1) driver's license suspension, such as for drink driving; 2) drivers with a disability certificate; and 3) drivers of luxury cars were excluded because a luxury car may indicate a higher socioeconomic status, and thus, other drivers may avoid directing their aggressive driving behavior towards such individuals. The retail price of the luxury motor vehicle was $>1.3$ million RMB, because the car retail price $>1.3$ million RMB was defined as a luxury car by China's Taxation Bureau. ${ }^{63}$

\section{Measures}

Social demographic data were collected, including gender, age, driving experience, and education. Gender was defined as male or female. Age was divided into four groups: [18-30] years old, [30-40] years old, [40-50] years old, and equal to or greater than 50 years old. Driving experience was divided into three groups: equal to or less than two years, 3-4 years, and equal to or more than five years. Education was divided into three groups: below senior high school (including senior high school), college education, and graduate school or above.

The aggressive driving behavior questionnaire developed by Luoyong was used in the current study. The questionnaire contains 23 items that assessed the following five factors: neglecting others, rapid lane changing, highspeed driving, risky overtaking, and personal assailment (noted as an Appendix) ${ }^{64}$ Neglecting others, such as not using the signal light when turning, was included. Rapid lane changing meant the driver moves his or her vehicle into a different lane rapidly. High speed driving referred to driving too fast disregarding speed limits. Personal assailment indicated hostility behaviors referred to as abusing or glaring at other drivers. Each factor was assessed on both A and B part. The A scale indicated real aggressive driving experience in certain conditions. B scale indicated cognitive control ability in terms of aggressive driving behavior when confronted by the same condition. Each question was scored on a five-point Likert scale. The test-retest reliability of each dimension of the scale ranges from $0.84-0.93$ and the Cronbach a coefficient ranges from $0.76-0.92 .{ }^{64}$ The total score for Part A, used in this study, assessed aggressive driving behavior. The higher the summary score for the A scale, the more likely the respondent drives aggressively.

The Generalized Anxiety Disorder (GAD-7) scale was used to assess the anxiety symptoms of participants. The GAD-7 is a seven-item, self-reported anxiety measurement tool designed by Spitzer. ${ }^{65}$ The total score is a sum of seven categories; the total score ranges from 0-21 with 0 4 indicating no GAD, 5-9 indicating mild GAD, 10-14 indicating moderate $\mathrm{GAD}$, and $15-21$ indicating severe GAD. The internal consistency coefficient of the GAD-7 is reported as 0.907 and the retest reliability coefficient is 0.413 , indicating that it has good reliability and validity. ${ }^{66}$

The Patient Health Questionnaire-9 (PHQ-9), evaluated depressive symptoms experienced during the preceding two weeks. There are nine items in this questionnaire. A total score between $0-4$ points indicates no depression, 5-9 indicates slight depression, 10-14 indicates moderate depression, 15-19 indicates moderately severe depression, and 20-27 indicates severe depression. ${ }^{67}$ The Chinese version of the PHQ-9 has been reported to have a Cronbach's $\alpha$ coefficient of $0.86 .^{68}$

The typical definitions of personality share the understanding that it includes sustainable patterns of feelings, thoughts, and actions that emerge with stability over time and with context. ${ }^{46}$ The Eysenck Personality Questionnaire-Short Scale (EPQ-RSC) was used to assess personality. The psychoticism, extraversion, and neuroticism model ( $\mathrm{P}-\mathrm{E}-\mathrm{N}$ model) of personality was developed by Eysenck. Critics of the psychoticism trait have suggested that it was too heterogeneous to be considered a single trait. The psychoticism (P) scale of the EPQ had provoked much criticism regarding its clinical value and inferior psychometric properties. Participants with mental disorders were excluded from this study. Thus, only the extraversion (E) and neuroticism (N) subscales of the EPQ-RSC were used in this study. The reliability and validity of the E scale were reported to be 0.85 and 0.64 , 
respectively. The reliability and validity of the $\mathrm{N}$ scale were 0.82 and 0.63 , respectively. ${ }^{69,70}$

Sleep is a naturally recurring rest-state of mind and body, characterized by relatively inhibited sensory activity, inhibition of nearly all voluntary muscles and distinguished from wakefulness by a decreased ability to react to stimuli. The Pittsburgh Sleep Quality Index (PSQI) was used to assess sleep quality. The PSQI was divided into seven factors, with the total score ranging from $0-21 .^{71}$ The higher the score, the lower the sleep quality. The psychometrics of the PSQI in insomnia patients was fittable. ${ }^{71}$ The Chinese version of the PSQI in the community ranks adults with insomnia an overall reliability coefficient of $0.82-0.83 .^{72}$

Abuse of illegal drugs is prohibited in China and is subject to legal constraints. Driving after drinking alcohol and driving while intoxicated in China is punished by driving-license suspension. Any driver whose driving license was suspended was excluded from this study. However, the drinking culture is prevalent in China and it was reported that alcohol use disorders would influence driving behavior. ${ }^{47,48}$ Therefore this article only considers the control of alcohol dependence. The Alcohol Use Disorders Identification Test (AUDIT), developed by the World Health Organization (WHO) in 1982, was used to screen for dangerous and harmful drinking. ${ }^{73}$ The scale consists of 10 items, with a score of 8 being positive for harmful drinking. The Cronbach's $\alpha$ coefficient for the Chinese Version of this AUDIT is reported to be 0.782 and the Chinese version of the AUDIT scale has been found to have better reliability and validity than the English version. ${ }^{74}$

Social support is where assistance is available from other people when needed, usually including emotional support, ${ }^{75}$ companionship support, ${ }^{76}$ informational support, ${ }^{77}$ and tangible assistance. ${ }^{78,79}$ Social support was measured by the Social Support Rating Scale (SSRS), formulated by Chinese scholar Xiao in 1986. The scale has ten items measuring three factors: objective support, subjective support, and social support utilization, with an overall Cronbach's $\alpha$ coefficient of 0.833 . The higher the score, the higher the level of social support. ${ }^{80}$

\section{Procedure}

The Ethics Committee of Hainan Medical University approved the cross-sectional study protocol. A crosssectional survey was designed and conducted between 1st February and 30th April 2019. A teacher with a Ph.D. in clinical psychology supervised a team of researchers comprising another two teachers and three undergraduates who majored in psychology. This team of researchers conducted the survey in Mandarin. Posters were put up at the Vehicle Management Office in the Yixing area to recruit participants to the study. The undergraduates took turns recruiting participants at the office. The participants were informed that it was an anonymous survey and that all answers would be kept strictly confidential and only used for research purposes. All participants were informed about the purpose of this study, in accordance with the Declaration of Helsinki. The survey was conducted with the participant's consent. Participants who consented to participate and met the inclusion and exclusion criteria completed the online survey for data collection.

\section{Statistical Analysis}

Descriptive statistics were performed using the SPSS24.0 software. The data were expressed as a number, percentage, and sub-group comparisons.The regression analysis on the influencing factors of aggressive driving behavior was undertaken using Jamovi statistical software and the simple slop test of interactions was automatically generated. Second, the SPSS macro program was also used to calculate the simple effect. Two kinds of methodology were used to determine the simple effect of the interaction.

\section{Results}

\section{Social Demographic Characteristics of the Sample}

Table 1 showed the social demographic characteristics of the sample. The sample comprised 529 participants, including 247 males (46.69\%) and 282 females (53.31\%). The majority of participants $(41.97 \%)$ were aged 18-30 years. Most of the participants had more than five years of driving experience $(39.32 \%)$ and most had a college education $(76.75 \%)$.

\section{Effects of Age and Driving Experience on the Dimensions of Aggressive Driving}

\section{Behavior}

Table 2 showed the analysis of aggressive driving behaviors of different driving-experience in the four dimensions and the subgroup analysis of different ages in each dimension. There were significant differences in the total score of neglecting others, rapid lane changing, risky overtaking, and personal assailment. 
Table I Collation and Analysis of Participants Demographic Information

\begin{tabular}{|c|c|c|c|}
\hline Variable & Category & Number of People & Percentage (\%) \\
\hline \multirow[t]{2}{*}{ Gender } & Male & 247 & 46.69 \\
\hline & Female & 282 & 53.31 \\
\hline \multirow[t]{4}{*}{ Age } & {$[18,30]$} & 222 & 41.97 \\
\hline & {$[30,40]$} & 135 & 25.52 \\
\hline & {$[40,50]$} & 140 & 26.47 \\
\hline & Over 50 & 32 & 6.05 \\
\hline \multirow[t]{3}{*}{ Driving experience } & $\leq 2$ years & 180 & 34.03 \\
\hline & $3-4$ years & $|4|$ & 26.65 \\
\hline & $\geq 5$ years & 208 & 39.32 \\
\hline \multirow[t]{3}{*}{ Educational level } & $\leq$ Senior high school & 101 & 19.09 \\
\hline & College education & 406 & 76.75 \\
\hline & Graduate school or above & 22 & 4.16 \\
\hline
\end{tabular}

Table 2 F-Test of Effects of Age and Driving-Experience on Aggressive Driving Behaviors

\begin{tabular}{|c|c|c|c|c|c|}
\hline \multirow[t]{2}{*}{ Factors } & \multicolumn{3}{|c|}{ Driving Experience } & \multirow[t]{2}{*}{$F$ Test Value } & \multirow[t]{2}{*}{$P$} \\
\hline & $\leq 2$ Years & 3-4 Years & $\geq 5$ Years & & \\
\hline Neglecting others & $9.26 \pm 0.35$ & $9.50 \pm 0.37$ & $7.66 \pm 0.23$ & 10.82 & 0.00 \\
\hline$[18,30]$ & $9.70 \pm 0.41$ & $10.61 \pm 0.563$ & $8.20 \pm 0.85$ & 1.86 & 0.16 \\
\hline$[30,40]$ & $7.37 \pm 0.73$ & $9.27 \pm 0.6 \mathrm{I}$ & $8.18 \pm 0.39$ & 2.56 & 0.08 \\
\hline$[40,50]$ & $8.38 \pm 1.75$ & $6.82 \pm 0.63$ & $7.16 \pm 0.32$ & 0.63 & 0.53 \\
\hline$\geq 50$ & $8.50 \pm 1.85$ & $8.80 \pm 1.39$ & $8.35 \pm 0.83$ & 0.03 & 0.97 \\
\hline Rapid lane changing & $9.56 \pm 0.37$ & $9.92 \pm 0.44$ & $7.12 \pm 0.23$ & 21.64 & 0.00 \\
\hline$[18,30]$ & $10.17 \pm 0.43$ & $\mid 1.21 \pm 0.69$ & $9.33 \pm 1.12$ & 1.24 & 0.29 \\
\hline$[30,40]$ & $7.48 \pm 0.83$ & $9.69 \pm 0.72$ & $7.82 \pm 0.54$ & 3.02 & 0.05 \\
\hline$[40,50]$ & $7.50 \pm 1.02$ & $7.00 \pm 0.67$ & $6.45 \pm 0.21$ & 1.12 & 0.33 \\
\hline$\geq 50$ & $6.75 \pm 0.75$ & $8.00 \pm 1.79$ & $7.09 \pm 0.722$ & 0.30 & 0.74 \\
\hline High speed driving & $9.58 \pm 0.36$ & $9.72 \pm 0.37$ & $10.27 \pm 0.35$ & 1.13 & 0.32 \\
\hline$[18,30]$ & $10.01 \pm 0.42$ & $\mid 1.21 \pm 0.69$ & $9.13 \pm 0.97$ & 0.72 & 0.46 \\
\hline$[30,40]$ & $7.67 \pm 0.68$ & $8.81 \pm 0.60$ & $10.43 \pm 0.7 \mid$ & 3.59 & 0.03 \\
\hline$[40,50]$ & $9.00 \pm 1.45$ & $8.86 \pm 0.84$ & $10.18 \pm 0.48$ & 0.84 & 0.43 \\
\hline$\geq 50$ & $8.50 \pm 1.50$ & $9.20 \pm 1.93$ & $11.04 \pm 1.13$ & 0.60 & 0.55 \\
\hline Risky overtaking & $12.38 \pm 0.44$ & $13.60 \pm 0.48$ & $11.56 \pm 0.33$ & 5.98 & 0.00 \\
\hline$[18,30]$ & $12.98 \pm 0.52$ & $15.62 \pm 0.75$ & $14.00 \pm 2.00$ & 4.01 & 0.02 \\
\hline$[30,40]$ & $10.26 \pm 0.99$ & $12.60 \pm 0.69$ & $11.90 \pm 0.63$ & 1.99 & 0.14 \\
\hline$[40,50]$ & $10.50 \pm 1.05$ & $10.18 \pm 0.85$ & $10.87 \pm 0.38$ & 0.30 & 0.74 \\
\hline$\geq 50$ & $9.25 \pm 1.18$ & $11.4 \pm 2.02$ & $12.35 \pm 0.92$ & 0.92 & 0.41 \\
\hline Personal assailment & $7.8 I \pm 0.28$ & $8.76 \pm 0.34$ & $7.39 \pm 0.25$ & 5.71 & 0.00 \\
\hline$[18,30]$ & $8.10 \pm 0.33$ & $9.92 \pm 0.544$ & $9.47 \pm|.5|$ & 4.48 & 0.01 \\
\hline$[30,40]$ & $6.78 \pm 0.64$ & $8.38 \pm 0.50$ & $7.95 \pm 0.49$ & 1.75 & 0.18 \\
\hline$[40,50]$ & $7.63 \pm 1.12$ & $6.55 \pm 0.58$ & $6.83 \pm 0.28$ & 0.40 & 0.67 \\
\hline$\geq 50$ & $5.00 \pm 0.71$ & $6.80 \pm 1.46$ & $7.22 \pm 0.62$ & 1.00 & 0.38 \\
\hline Total score & $48.59 \pm 1.54$ & $51.48 \pm 1.68$ & $44 \pm 1.05$ & 7.31 & 0.00 \\
\hline
\end{tabular}

Subgroups of different ages showed that the 30-40 age group differed in neglecting others, rapid lane changing, high-speed driving and the 18-30 age group differed in risky overtaking and personal assailment.

\section{Post Hoc Analysis of Age Groups and Driving Experience in Each Dimension}

If the difference in Levene's test was statistically significant $(p<0.05)$, the hypothesis of homogeneity of variance 
was not satisfied. If the difference in Levene's test was not statistically significant ( $>0.05$ ), the hypothesis of homogeneity of variance could not be rejected. When the homogeneity of variance was met, the Tukey's test can then compare groups in pairs. When the homogeneity of variance was not met, the Games-Howell test was performed in pairs between groups.

The multiple factor comparisons of the $[18,30]$ age group within the different driving experience groups

As shown in Table 3, no significant difference was detected using Levene's test between high- speed driving, neglecting others, rapid lane changing and risky overtaking $(\mathrm{p}>0.05)$. As the variance was uniform, Tukey's tests were performed for group comparisons. Personal assailment was statistically significant in Levene's test $(\mathrm{p}<0.05)$, however, the variance was not uniform, and the GamesHowell test was performed.

Table 4 showed that when the driving experience was 3-4 years compared with that of $\leq 2$ years, there was

Table 3 Homogeneity of Variance Test

\begin{tabular}{|l|c|c|}
\hline Factors & Levene Statistic & $\mathbf{P}$ \\
\hline Neglecting others & 1.09 & 0.34 \\
Rapid lane changing & 0.57 & 0.56 \\
High speed driving & 0.91 & 0.41 \\
Risky overtaking & 0.54 & 0.59 \\
Personal assailment & 3.74 & 0.03 \\
\hline
\end{tabular}

a significant difference in the dimension of risky overtaking. When the driving experience was 3-4 years, compared with driving years $\leq 2$ years, there was a significant difference in the dimension of personal assailment.

The multiple factor comparisons of the $[30,40]$ age group within the different driving experience groups

As shown in Table 5, neglecting others, rapid lane changing, risky overtaking and personal assailment showed no statistically significant difference using Levene's test $(p>0.05)$. As the variance was uniform, Tukey's tests were performed and high-speed driving was statistically significant using Levene's test $(p<0.05$ ), then the variance was not uniform, and the Games-Howell test was performed.

Table 6 showed that when the driving experience was 3-4 years compared with that of $\leq 2$ years, there was a significant difference in the dimension of neglecting others. When driving years $\geq 5$ years, there was a significant difference in the dimension of rapid lane changing compared with driving years 3-4 years. When driving years $\geq 5$ years, compared with driving years $\leq 2$ years, there was a significant difference in the dimension of high speed driving.

The multiple factor comparisons of the $[40,50]$ age group within the different driving experience groups

As shown in Table 7, no statistically significant difference in each dimension was detected $(p>0.05)$. As all the variances were uniform, Tukey's tests were performed.

Table 4 Group Comparison in the [18, 30] Age Group

\begin{tabular}{|c|c|c|c|c|c|c|c|c|}
\hline & \multirow[t]{2}{*}{ Way } & \multirow[t]{2}{*}{ DE (I) } & \multirow[t]{2}{*}{ DE (J) } & \multirow{2}{*}{ MD (I- J) } & \multirow[t]{2}{*}{ SE } & \multirow[t]{2}{*}{$\mathbf{P}$} & \multicolumn{2}{|c|}{$95 \% \mathrm{Cl}$} \\
\hline & & & & & & & LB & UB \\
\hline \multirow[t]{3}{*}{ Neglecting others } & \multirow[t]{3}{*}{$\mathrm{T}$} & $\leq 2$ years & $3-4$ years & -0.91 & 0.70 & 0.40 & -2.56 & 0.74 \\
\hline & & $\leq 2$ years & $\geq 5$ years & 1.50 & 1.27 & 0.47 & $-1.5 \mid$ & 4.50 \\
\hline & & $3-4$ years & $\geq 5$ years & 2.41 & 1.34 & 0.17 & -0.76 & 5.57 \\
\hline \multirow[t]{3}{*}{ Rapid lane changing } & \multirow[t]{3}{*}{$\mathrm{T}$} & $\leq 2$ years & $3-4$ years & -1.04 & 0.78 & 0.38 & -2.88 & 0.80 \\
\hline & & $\leq 2$ years & $\geq 5$ years & 0.84 & 1.42 & 0.83 & -2.51 & 4.19 \\
\hline & & $3-4$ years & $\geq 5$ years & 1.88 & 1.49 & 0.42 & -1.65 & 5.41 \\
\hline \multirow[t]{3}{*}{ High speed driving } & \multirow[t]{3}{*}{$\mathrm{T}$} & $\leq 2$ years & $3-4$ years & -0.65 & 0.72 & 0.64 & -2.34 & 1.04 \\
\hline & & $\leq 2$ years & $\geq 5$ years & 0.88 & 1.31 & 0.78 & -2.20 & 3.96 \\
\hline & & $3-4$ years & $\geq 5$ years & 1.53 & 1.38 & 0.51 & -1.71 & 4.78 \\
\hline \multirow[t]{3}{*}{ Risky overtaking } & \multirow[t]{3}{*}{$\mathrm{T}$} & $\leq 2$ years & $3-4$ years & -2.64 & 0.93 & 0.01 & -4.85 & -0.44 \\
\hline & & $\leq 2$ years & $\geq 5$ years & -1.02 & 1.70 & 0.82 & -5.03 & 2.99 \\
\hline & & $3-4$ years & $\geq 5$ years & 1.62 & 1.79 & 0.64 & -2.60 & 5.85 \\
\hline \multirow[t]{3}{*}{ Personal assailment } & \multirow[t]{3}{*}{$\mathrm{G}-\mathrm{H}$} & $\leq 2$ years & $3-4$ years & -1.83 & 0.64 & 0.01 & -3.33 & -0.32 \\
\hline & & $\leq 2$ years & $\geq 5$ years & -1.37 & 1.54 & 0.66 & -5.36 & 2.62 \\
\hline & & $3-4$ years & $\geq 5$ years & 0.46 & 1.60 & 0.96 & -3.63 & 4.55 \\
\hline
\end{tabular}

Abbreviations: T, Tukey's test; G-H, Games-Howell test; DE, driving experience. 
Table 5 Homogeneity of Variance Test

\begin{tabular}{|l|c|c|}
\hline Factors & Levene Statistic & P \\
\hline Neglecting others & 2.64 & 0.08 \\
Rapid lane changing & 1.93 & 0.15 \\
High-speed driving & 4.45 & 0.01 \\
Risky overtaking & 0.22 & 0.80 \\
Personal assailment & 0.11 & 0.90 \\
\hline
\end{tabular}

Table 8 shows that in the group [40, 50] years old, there was no significant difference between each subgroup.

The multiple factor comparisons of equal to or over 50 years old age group within the different driving experience groups

As illustrated in Table 9, no statistically significant difference was detected for any dimensions $(\mathrm{p}>0.05)$. The variance was uniform, so Tukey's tests were performed.

Table 10 showed that when drivers were aged $\geq 50$ years old, there was no significant difference between each subgroup.

\section{Multiple Analyses of the Factors Influencing Aggressive Driving Behaviors}

Table 11 showed the regression analysis results testing the influences of various factors on aggressive driving behavior. Age and neuroticism were significant influencing factors, and the interaction between age, driving experience, and depression was statistically significant. Similarly, the interaction
Table 7 Homogeneity of Variance Test

\begin{tabular}{|l|c|c|}
\hline Factors & Levene Statistic & $\mathbf{P}$ \\
\hline Neglecting others & 2.68 & 0.07 \\
Rapid lane changing & 1.85 & 0.16 \\
High speed driving & 1.25 & 0.29 \\
Risky overtaking & 0.46 & 0.64 \\
Personal assailment & 0.47 & 0.63 \\
\hline
\end{tabular}

between age, driving experience, and neuroticism was statistically significant. Multicollinearity first proposed by Frisch, ${ }^{81}$ refers to the correlations between two or more dependent variables in a linear regression model, influencing the associations between dependent and independent variables. Variance inflation factor (VIF) is a measure of collinearity in a multiple linear regression model. Quinn and Keough suggested that when $\mathrm{VIF}<10$, there was no obvious multicollinearity. ${ }^{82}$ In statistical analysis, when $10 \leq \mathrm{VIF}<100$, the inference is that obvious multicollinearity exists, and there is severe multicollinearity when $\mathrm{VIF} \geq 100$. All VIF values were tested and found to be in the acceptable range.

\section{Simple Effects of Age, Driving Experience, and Depressive Symptoms}

Regression analysis showed that the interaction between age, driving experience, and depression was significant, so a simple slope test was conducted to characterize the interaction. Firstly, the Jamovi analysis automatic classification program was used to calculate

Table 6 Group Comparison in the [30, 40] Age Group

\begin{tabular}{|c|c|c|c|c|c|c|c|c|}
\hline & \multirow[t]{2}{*}{ Way } & \multirow[t]{2}{*}{ DE (I) } & \multirow[t]{2}{*}{ DE (J) } & \multirow[t]{2}{*}{ MD (I- J) } & \multirow[t]{2}{*}{ SE } & \multirow[t]{2}{*}{$\mathbf{P}$} & \multicolumn{2}{|c|}{$95 \% \mathrm{Cl}$} \\
\hline & & & & & & & LB & UB \\
\hline \multirow[t]{3}{*}{ Neglecting others } & $\mathrm{T}$ & $\leq 2$ years & $3-4$ years & -1.90 & 0.88 & 0.08 & -3.98 & 0.18 \\
\hline & & $\leq 2$ years & $\geq 5$ years & -0.813 & 0.84 & 0.60 & -2.81 & 1.19 \\
\hline & & $3-4$ years & $\geq 5$ years & 1.088 & $0.7 I$ & 0.28 & -0.58 & 2.76 \\
\hline \multirow[t]{3}{*}{ Rapid lane changing } & $\mathrm{T}$ & $\leq 2$ years & $3-4$ years & -2.21 & 1.09 & 0.11 & -4.78 & 0.37 \\
\hline & & $\leq 2$ years & $\geq 5$ years & -0.34 & 1.05 & 0.95 & -2.81 & 2.14 \\
\hline & & $3-4$ years & $\geq 5$ years & 1.87 & 0.87 & 0.09 & -0.20 & 3.94 \\
\hline \multirow[t]{3}{*}{ High speed driving } & $\mathrm{G}-\mathrm{H}$ & $\leq 2$ years & $3-4$ years & -1.15 & 0.91 & 0.43 & -3.34 & 1.04 \\
\hline & & $\leq 2$ years & $\geq 5$ years & -2.77 & 0.99 & 0.02 & -5.13 & -0.40 \\
\hline & & $3-4$ years & $\geq 5$ years & -1.62 & 0.93 & 0.20 & -3.84 & 0.60 \\
\hline \multirow[t]{3}{*}{ Risky overtaking } & $\mathrm{T}$ & $\leq 2$ years & $3-4$ years & -2.35 & 1.18 & 0.12 & -5.14 & 0.45 \\
\hline & & $\leq 2$ years & $\geq 5$ years & -1.64 & 1.14 & 0.32 & -4.33 & 1.05 \\
\hline & & $3-4$ years & $\geq 5$ years & 0.70 & 0.95 & 0.74 & -1.54 & 2.95 \\
\hline \multirow[t]{3}{*}{ Personal assailment } & G-H & $\leq 2$ years & $3-4$ years & -1.60 & 0.86 & 0.16 & -3.64 & 0.44 \\
\hline & & $\leq 2$ years & $\geq 5$ years & -1.17 & 0.83 & 0.34 & -3.14 & 0.79 \\
\hline & & $3-4$ years & $\geq 5$ years & 0.43 & 0.69 & 0.81 & -1.22 & 2.07 \\
\hline
\end{tabular}


Table 8 Group Comparison in the [40, 50] Age Group

\begin{tabular}{|c|c|c|c|c|c|c|c|c|}
\hline & \multirow{2}{*}{ Way } & \multirow{2}{*}{ DE (I) } & \multirow{2}{*}{ DE (J) } & \multirow{2}{*}{ MD (I- J) } & \multirow[t]{2}{*}{ SE } & \multirow[t]{2}{*}{$\mathbf{P}$} & \multicolumn{2}{|c|}{$95 \% \mathrm{Cl}$} \\
\hline & & & & & & & LB & UB \\
\hline \multirow[t]{3}{*}{ Neglecting others } & \multirow[t]{3}{*}{$\mathrm{T}$} & $\leq 2$ years & $3-4$ years & 1.56 & 1.39 & 0.51 & -1.74 & 4.86 \\
\hline & & $\leq 2$ years & $\geq 5$ years & 1.21 & 1.24 & 0.59 & -1.71 & 4.14 \\
\hline & & $3-4$ years & $\geq 5$ years & -0.35 & 0.79 & 0.90 & -2.21 & 1.52 \\
\hline \multirow[t]{3}{*}{ Rapid lane changing } & \multirow[t]{3}{*}{$\mathrm{T}$} & $\leq 2$ years & $3-4$ years & 0.50 & 0.98 & 0.87 & -1.82 & 2.82 \\
\hline & & $\leq 2$ years & $\geq 5$ years & 1.06 & 0.87 & 0.45 & -1.01 & 3.12 \\
\hline & & $3-4$ years & $\geq 5$ years & 0.56 & 0.56 & 0.58 & -0.76 & 1.87 \\
\hline \multirow[t]{3}{*}{ High speed driving } & \multirow[t]{3}{*}{$\mathrm{T}$} & $\leq 2$ years & $3-4$ years & 0.14 & 1.97 & 0.99 & -4.57 & 4.84 \\
\hline & & $\leq 2$ years & $\geq 5$ years & -1.18 & 1.76 & 0.78 & -5.35 & 2.99 \\
\hline & & $3-4$ years & $\geq 5$ years & -1.32 & 1.12 & 0.47 & -3.98 & 1.34 \\
\hline \multirow[t]{3}{*}{ Risky overtaking } & \multirow[t]{3}{*}{$\mathrm{T}$} & $\leq 2$ years & $3-4$ years & 0.32 & 1.61 & 0.98 & -3.50 & 4.13 \\
\hline & & $\leq 2$ years & $\geq 5$ years & -0.37 & 1.43 & 0.96 & -3.76 & 3.01 \\
\hline & & $3-4$ years & $\geq 5$ years & -0.69 & 0.91 & 0.73 & -2.85 & 1.47 \\
\hline \multirow[t]{3}{*}{ Personal assailment } & \multirow[t]{3}{*}{$\mathrm{T}$} & $\leq 2$ years & $3-4$ years & 1.08 & 1.21 & 0.65 & -1.80 & 3.96 \\
\hline & & $\leq 2$ years & $\geq 5$ years & 0.80 & 1.08 & 0.74 & -1.75 & 3.35 \\
\hline & & $3-4$ years & $\geq 5$ years & -0.28 & 0.69 & 0.91 & -1.91 & 1.35 \\
\hline
\end{tabular}

Table 9 Homogeneity of Variance Test

\begin{tabular}{|l|c|c|}
\hline Factors & Levene Statistic & $\mathbf{P}$ \\
\hline Neglecting others & 0.24 & 0.79 \\
Rapid lane changing & 0.48 & 0.62 \\
High speed driving & 0.65 & 0.53 \\
Risky overtaking & 0.40 & 0.67 \\
Personal assailment & 0.99 & 0.39 \\
\hline
\end{tabular}

the interaction. The classification program divided depressive symptoms into three levels: the average minus one standard deviation level, the average level, and the average plus one standard deviation level. The result of this analysis was shown in Figure 1. The top two panels showed that for those with depressive symptoms one standard deviation less than the mean and those with the mean level depression symptoms, aggressive driving behaviors decreased with age and experience. However, the third panel showed that older and experienced drivers were more likely to engage in aggressive driving behavior when experiencing more severe depressive symptoms.

The SPSS macro program was also used to calculate the simple effect of the interactions. According to the PHQ-9 cut-off classification, in the absence of depressive symptoms (PHQ-9 $\leqq 4$ ), the overall trend in aggressive driving behavior decreased with increasing age and driving experience (Figure 2). As shown in Figure 3, in the case of mild depressive symptoms ( $5 \leqq$ PHQ- $9 \leqq 9$ ), when participants' were aged older than 40 , regardless of driving experience levels, the occurrence of aggressive driving behavior demonstrated an upward trend. As shown in Figure 4, in the case of moderate depressive symptoms ( $10 \leqq$ PHQ- $9 \leqq 14)$, aggressive driving behavior showed a downward trend with increasing age and driving experience. As shown in Figure 5, in the case of

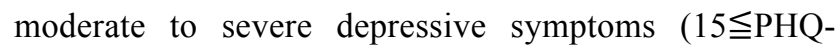
$9 \leqq 19$ ), the aggressive driving behavior of drivers older than 30 years old showed an upward trend. As shown in Figure 6, in the case of severe depressive symptoms ( $20 \leqq$ PHQ-9 927 ), the aggressive driving behavior of drivers with driving experience of more than 5 years and aged greater than 30 years showed an increasing trend.

\section{Simple Effect Test of Age, Driving Experience, and Neuroticism}

The interaction effect of age, driving experience, and neuroticism was statistically significant, so a further simple effect test was conducted for this interaction. Firstly, the Jamovi analysis automatic classification program was used to calculate the interaction. The classification program divided neuroticism into three levels: average minus one standard deviation level, average level, and average plus one standard deviation level. The results were shown in Figure 7. The Jamovi simple effect test showed that the total tendency was aggressive driving behavior decreased with increasing 
Table 10 Group Comparison in the $\geq 50$ Years Old Group

\begin{tabular}{|c|c|c|c|c|c|c|c|c|}
\hline & \multirow[t]{2}{*}{ Way } & \multirow[t]{2}{*}{ DE (I) } & \multirow[t]{2}{*}{ DE (J) } & \multirow[t]{2}{*}{ MD (I- J) } & \multirow[t]{2}{*}{ SE } & \multirow[t]{2}{*}{$\mathbf{P}$} & \multicolumn{2}{|c|}{$95 \% \mathrm{Cl}$} \\
\hline & & & & & & & LB & UB \\
\hline \multirow[t]{3}{*}{ Neglecting others } & $\mathrm{T}$ & $\leq 2$ years & $3-4$ years & -0.30 & 2.59 & 0.99 & -6.69 & 6.09 \\
\hline & & $\leq 2$ years & $\geq 5$ years & 0.15 & 2.09 & 0.99 & -5.01 & 5.31 \\
\hline & & $3-4$ years & $\geq 5$ years & 0.45 & 1.90 & 0.97 & -4.25 & 5.15 \\
\hline \multirow[t]{3}{*}{ Rapid lane changing } & $\mathrm{T}$ & $\leq 2$ years & $3-4$ years & -1.75 & 2.28 & 0.73 & -7.38 & 3.88 \\
\hline & & $\leq 2$ years & $\geq 5$ years & -0.84 & 1.84 & 0.89 & -5.38 & 3.71 \\
\hline & & $3-4$ years & $\geq 5$ years & 0.91 & 1.68 & 0.85 & -3.23 & 5.05 \\
\hline \multirow[t]{3}{*}{ High speed driving } & $\mathrm{T}$ & $\leq 2$ years & $3-4$ years & -0.70 & $3.4 I$ & 0.98 & -9.12 & 7.72 \\
\hline & & $\leq 2$ years & $\geq 5$ years & -2.54 & 2.75 & 0.63 & -9.34 & 4.25 \\
\hline & & $3-4$ years & $\geq 5$ years & -1.84 & 2.51 & 0.75 & -8.04 & 4.35 \\
\hline \multirow[t]{3}{*}{ Risky overtaking } & $\mathrm{T}$ & $\leq 2$ years & $3-4$ years & -2.15 & 2.87 & 0.74 & -9.23 & 4.93 \\
\hline & & $\leq 2$ years & $\geq 5$ years & -3.10 & 2.31 & 0.39 & -8.81 & 2.62 \\
\hline & & $3-4$ years & $\geq 5$ years & -0.95 & 2.11 & 0.90 & -6.15 & 4.26 \\
\hline \multirow[t]{3}{*}{ Personal assailment } & $\mathrm{T}$ & $\leq 2$ years & $3-4$ years & -1.80 & 1.94 & 0.63 & -6.59 & 2.99 \\
\hline & & $\leq 2$ years & $\geq 5$ years & -2.22 & 1.57 & 0.35 & -6.09 & 1.65 \\
\hline & & $3-4$ years & $\geq 5$ years & -0.42 & 1.43 & 0.95 & -3.94 & 3.11 \\
\hline
\end{tabular}

Table I I Regression Analysis of Factors Influencing Aggressive Driving

\begin{tabular}{|c|c|c|c|c|}
\hline Variable & Estimate & SE & $t$ & $P$ \\
\hline Intercept & 74.81 & 10.62 & 7.04 & 0.00 \\
\hline Age & -7.43 & 2.42 & -3.07 & 0.00 \\
\hline Gender & -4.46 & 2.57 & -1.73 & 0.08 \\
\hline Education & -2.46 & 2.93 & -0.84 & 0.40 \\
\hline Driving experience & -2.24 & 2.29 & -0.98 & 0.33 \\
\hline Depression symptoms & -0.67 & 0.36 & -1.87 & 0.06 \\
\hline Anxiety symptoms & 0.52 & 0.45 & 1.14 & 0.25 \\
\hline Extraversion & -0.47 & 0.45 & -1.04 & 0.30 \\
\hline Neuroticism & 1.01 & 0.41 & 2.46 & 0.01 \\
\hline Alcohol dependence & 0.26 & 0.23 & 1.16 & 0.25 \\
\hline Social support & -0.24 & 0.17 & -1.40 & 0.16 \\
\hline Sleep quality & 0.96 & 0.50 & 1.91 & 0.06 \\
\hline Age $*$ driving experience $*$ gender & -0.05 & 0.46 & -0.11 & 0.92 \\
\hline Age $*$ driving experience $*$ education & 0.44 & 0.46 & 0.96 & 0.34 \\
\hline Age $*$ driving experience $*$ depression & 0.20 & 0.08 & 2.38 & 0.02 \\
\hline Age $*$ driving experience $*$ anxiety & -0.07 & 0.10 & -0.74 & 0.46 \\
\hline Age $*$ driving experience $*$ extraversion & 0.07 & 0.08 & 0.95 & 0.34 \\
\hline Age $*$ driving experience $*$ neuroticism & -0.17 & 0.08 & -2.20 & 0.03 \\
\hline Age $*$ driving experience $*$ alcohol dependence & -0.03 & 0.05 & -0.51 & 0.61 \\
\hline Age $*$ driving experience $*$ social support & 0.00 & 0.02 & 0.10 & 0.92 \\
\hline Age $*$ driving experience $*$ sleep quality & 0.09 & 0.09 & 0.91 & 0.36 \\
\hline
\end{tabular}

age. However, shorter driving experience participants decreased much more sharply than more highly experienced drivers engaging in aggressive driving.

Then, using the SPSS macro program, the simple effects of age, driving years, and neuroticism (aboveaverage level, and below average level) were calculated.
As shown in Figures 8 and 9, the general trend showed that increasing age decreased aggressive driving behavior. Shorter driving experienced participants decreased much more obviously than longer experienced drivers in aggressive driving when experiencing both reduced and higher neuroticism. 


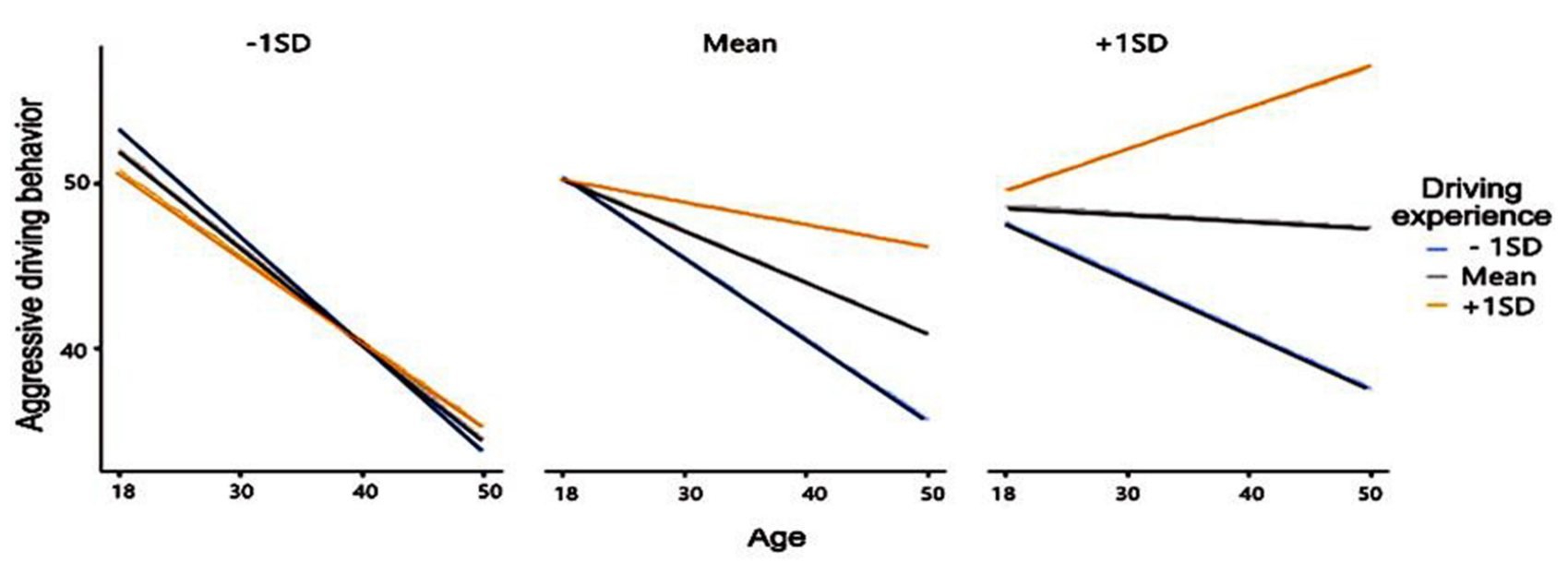

Figure I The simple effect of age, driving experience and depression symptoms using Jamovi software.

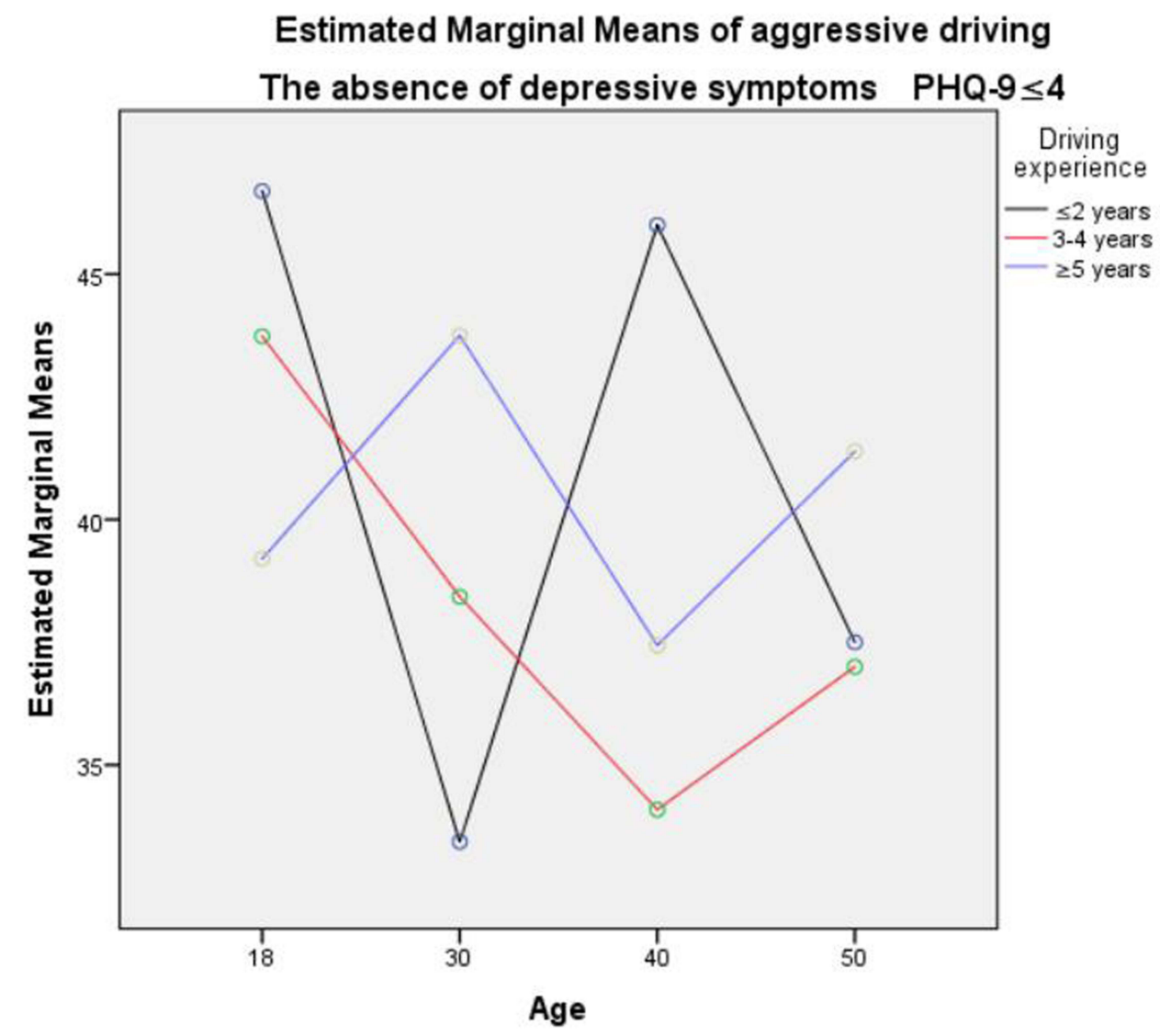

Figure 2 The simple effect of age, driving experience and depression symptoms when PHQ-9 $\leqq 4$ using SPSS.

\section{Discussion}

It has been previously reported that driver factors are directly or indirectly responsible for approximately $90 \%$ of traffic accidents. ${ }^{25,26,83,84}$ The recognition of risky drivers prone to aggressive driving behaviors and accident involvement is essential for ensuring traffic and public safety. It has previously been asserted that drivers' selfreporting accurately represents their actual behavior, ${ }^{85}$ especially when surveys were conducted anonymously, ${ }^{86}$ inferring that findings from this anonymous survey would be reliable. Gender was not a significant impact of aggressive driving behavior probably because the female sample 


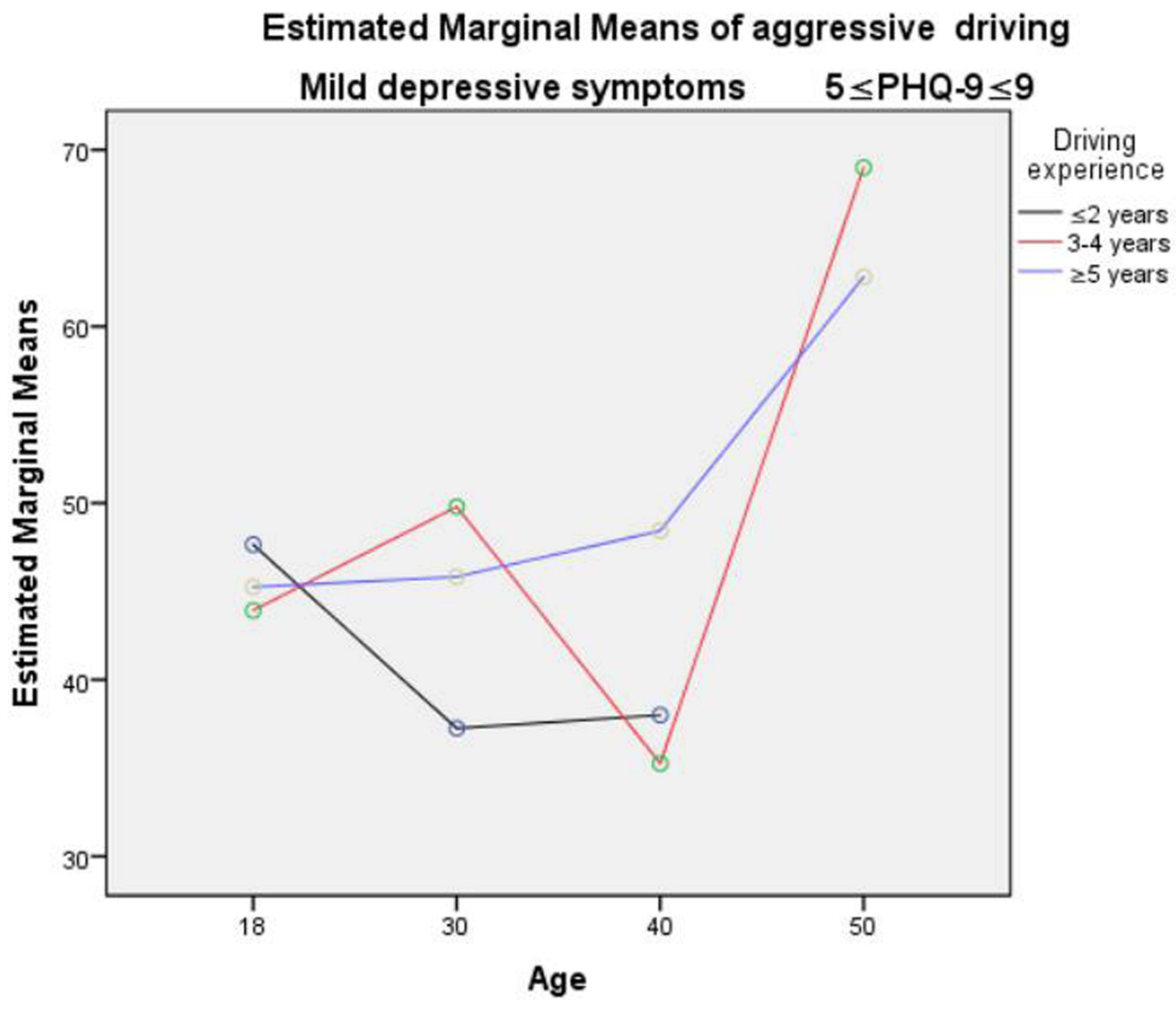

Non-estimable means are not plotted

Figure 3 The simple effect of age, driving experience and depressive symptoms when $5 \leqq P H Q-9 \leqq 9$ using SPSS.

was slightly higher than the male sample in an insufficiently sized sample pool.

\section{Age and Aggressive Driving Behavior}

Drivers of different ages may exhibit distinct cognition, driving abilities and risk preferences in any given traffic environment. The results from the current study elucidated that age and aggressive driving behavior was negatively correlated, consistent with previous studies that elderly drivers were safer drivers and in comparison, younger drivers were prone to commit increasingly risk-taking driving. ${ }^{26,27,87}$ However, the above studies ignored that other variables interacted with age during global traffic environment. Zhang analyzed the interaction of age and sex on aggression, and found the converse trend that aggressive driving increased with age. ${ }^{28}$ Guo analyzed the interaction of age and personality traits finding that elderly drivers would be more accident-involvement. ${ }^{29}$ These inconsistent findings from different scholars indicated that the influence of age on aggressive behavior would be more complicated when considering the interaction effects in natural traffic environments.
The interaction analysis from the current study indicated that veteran drivers with depressive symptoms could be more prone to aggressive driving behaviors. First, the Jamovi automatic analysis indicated that older drivers and those with more driving experience were more likely to show aggressive driving behaviors when experiencing higher depressive symptoms. Second, based on the PHQ-9 cut-off criterion, among those participants with mild depressive symptoms, older drivers and those with more driving experience were prone to aggressive driving behaviors. Together, these results derived using different assessment methods indicated that slight depressive symptoms increase the aggressive driving behaviors of older and more experienced drivers. In addition, the SPSS macro program showed that in the case of moderate to severe depressive symptoms, the aggressive driving behavior of drivers older than 30 years with all levels of driving experiences exhibited an upward trend. Moreover, when exhibiting severe depressive symptoms, the aggressive driving behavior of drivers with more than five years' experience and those aged greater than 30 years also showed an increasing trend. 


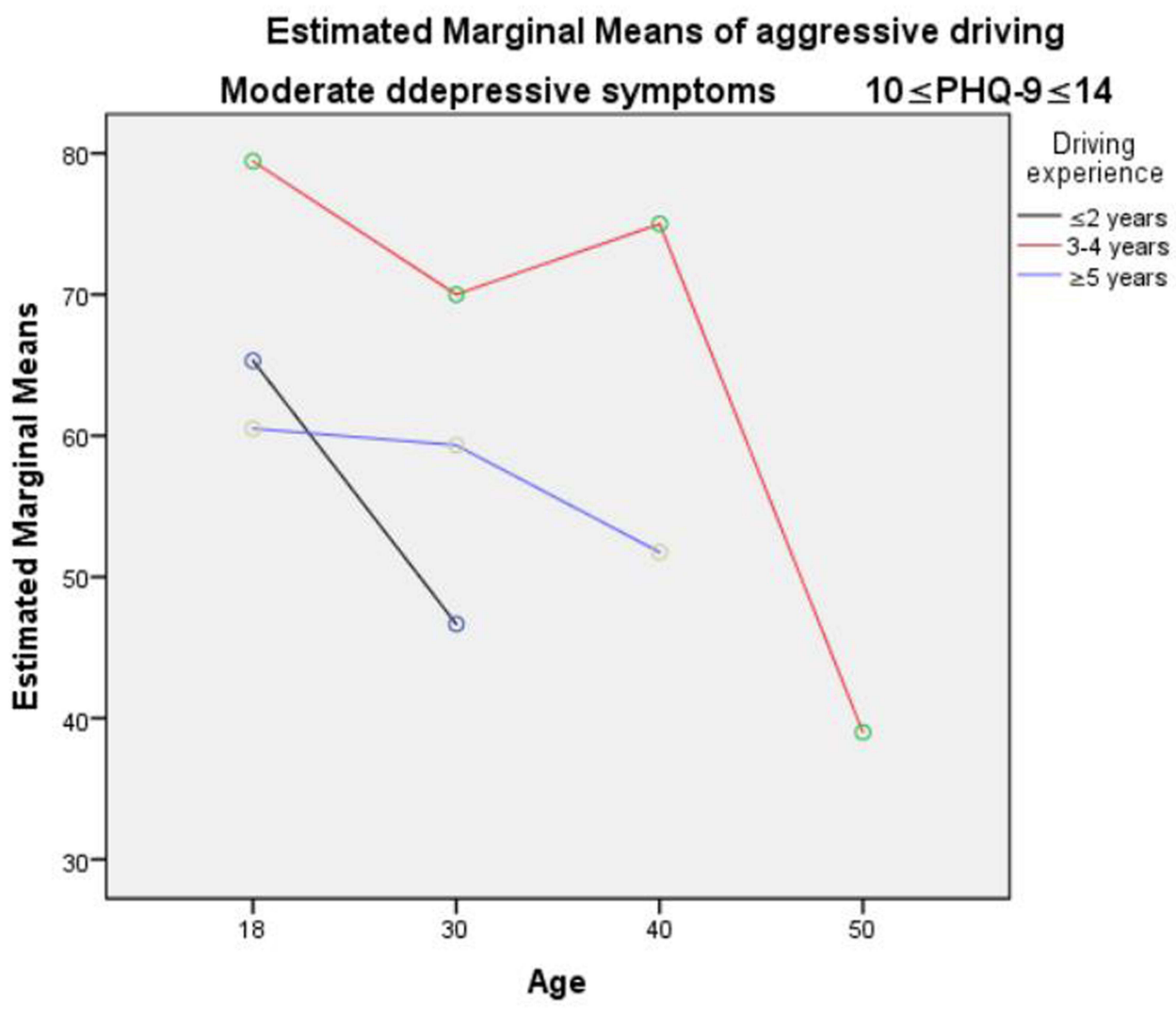

Non-estimable means are not plotted

Figure 4 The simple effect of age, driving experience and depression symptoms when $10 \leqq P H Q-9 \leqq 14$ using SPSS.

Associations between depression and aggression in children, adolescents ${ }^{88-91}$ and schizophrenic patients ${ }^{92}$ have been previously described. To the authors' knowledge, this study is the first to show an association between depressive symptoms and aggressive behavior in the general adult daily lives setting. Depressed individuals may potentially be prone to aggression due to depressive symptoms being correlated with irritable moods, ${ }^{93-95}$ and irritable moods were correlated with impulsivity, ${ }^{96,97}$ which was closely associated with aggression. ${ }^{98-100}$ Praag suggested that increased aggressive behaviors be thought of as the pacemakers of depression. ${ }^{101}$ The coinciding linkage of depression and aggression suggested a shared neuroendocrine pathway. This result indicated a clinical potential of anti-depressants for treatment of aggressive behavior, as previously discussed by other scholars. ${ }^{102-104}$

According to the current and previous studies, aggressive driving prevention among experienced adult drivers should consider treating depressive symptoms. Such treatment methods include physical treatments, medication, and psychotherapies. Physical therapy included electroconvulsive therapy (ETC), ${ }^{105}$ vagus nerve stimulation,,${ }^{106}$ and magnetic seizure therapy. ${ }^{107-109}$ Medications referred to traditional antidepressants and new antidepressants. The traditional clinical drugs mainly included tricyclic antidepressants, ${ }^{110}$ monoamine oxidase inhibitors (MAO1). ${ }^{111}$ The new type antidepressants mainly included selective serotonin reuptake inhibitors (SSRIs), ${ }^{112}$ and selective serotonin-norepinephrine reuptake inhibitors (selective serotonin-norepinephrine reuptake inhibitors, SNRIs). ${ }^{113}$ However, for those drivers who are not diagnosed with clinical depression, psychotherapy, ${ }^{114}$ would be safer and more practical to be administered by the traffic administration department. Internet cognitive behavioral therapy (iCBT) had been found effective for the treatment of depressive symptoms as well as other psychosomatic symptoms and psychosocial problems. ${ }^{115-118}$ In the COVID-19 background. iCBT could be a safe, cost-effective, feasible and immediately accessible method to moderate and mitigate the depressive symptoms for broadly targeted drivers. 


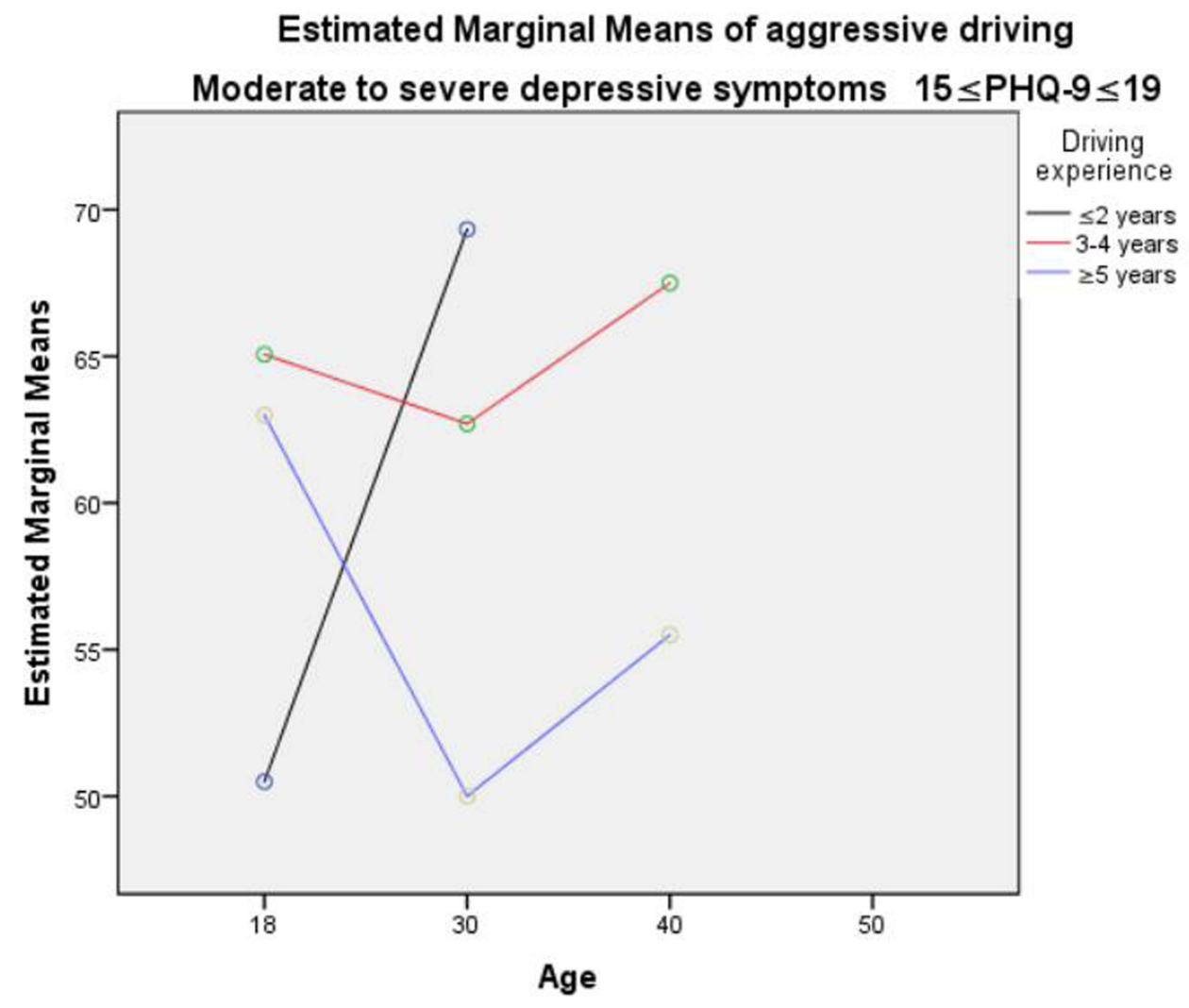

Non-estimable means are not plotted

Figure 5 The simple effect of age, driving experience and depression symptoms when $15 \leqq P H Q-9 \leqq 19$ using SPSS

\section{Neuroticism and Aggressive Driving Behavior}

Personality reveals individuals' behavioral patterns in a variety of situations. Personality traits may be stable and could predispose individuals' emotional arousal and behavioral responses. ${ }^{119}$ The multiple regression model of the data showed that neuroticism had a significantly positive correlation with aggressive driving. This finding was consistent with previous studies that neuroticism was associated with more accident involvement, traffic violation $^{29,120,121}$ and anti-social behavior. ${ }^{122}$

The psychological mechanism for the association between neuroticism and aggressive behavior probably is due to the following reasons. First, people experiencing neuroticism tend to experience negative emotions such as stress, depression, and shame. Individuals in higher neuroticism might be easily emotional angered or frustrated, which triggers aggressive driving. Further, people with high neuroticism tend to have emotional instability, which means they tend to respond impulsively to stimuli and be overly-emotional. Many a study found that individuals with emotional instability, such as sensitivity to express impulsivity, or high sensation seeking, can be predisposed to aggressive driving behaviors across conditions. $^{123-125}$ The neurocognitive mechanism of neuroticism and aggressive behavior is unclear. Barratt and Krakowski et al reported patients with frontal impairment could not inhibit their violent impulses. ${ }^{126,127}$ The instrumental anti-social aggression was suggested to be related to a lack of moral socialization and empathy due to a lack of development of the violence inhibition system. ${ }^{128-130}$ Whether specific neural cue for an inhibitory pathway can explain the association of neuroticism and aggression association deserves further exploration.

The SPSS simple effect test also showed that drivers with less driving experience decreased much more sharply than more highly experienced drivers in aggressive driving within both neuroticism levels. Such trends indicated that drivers with more extended driving experience who were experiencing neuroticism required more intensive follow-up investigation and interventions. To screen and identify individuals with high neurotic personality 


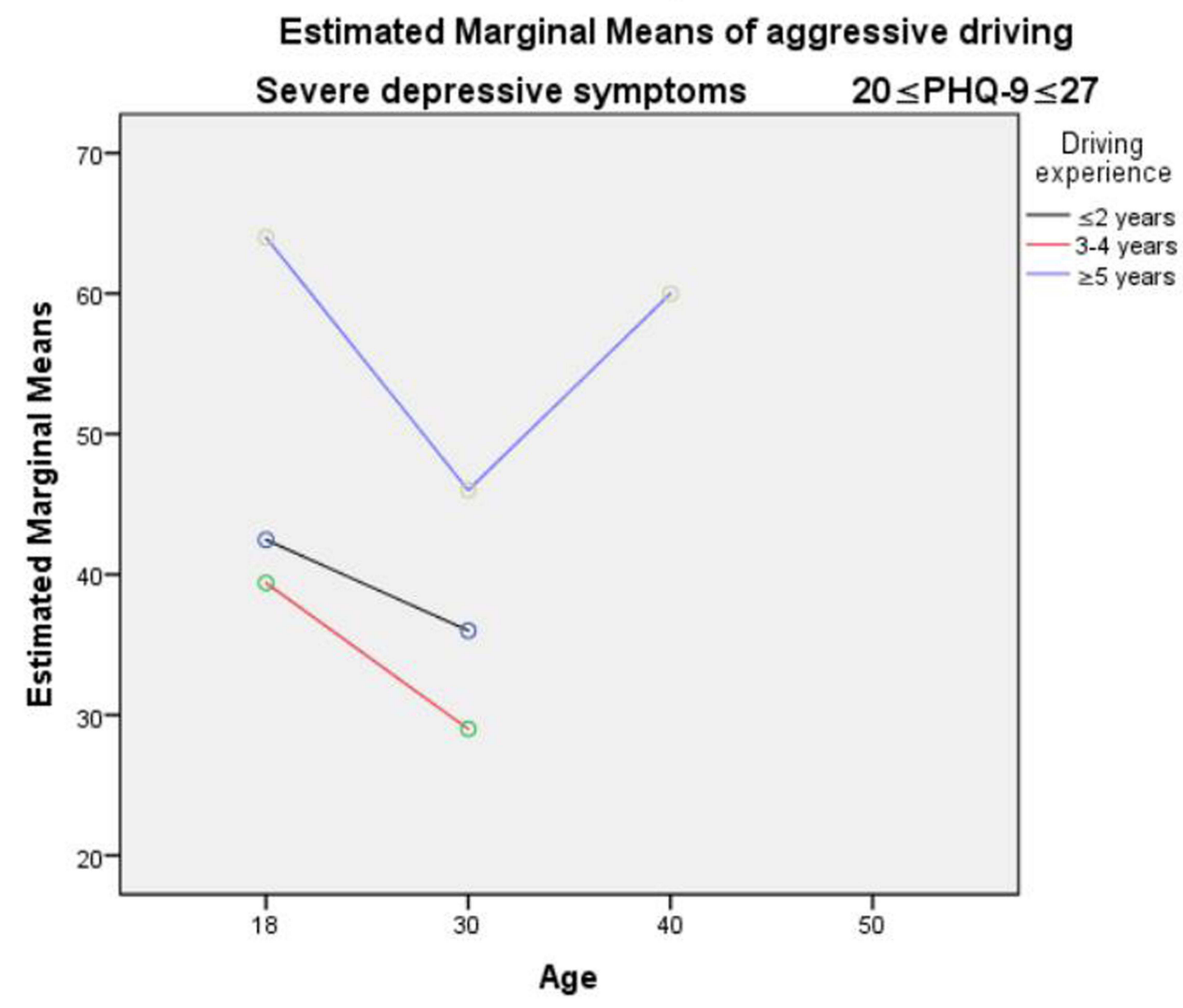

Non-estimable means are not plotted

Figure 6 The simple effect of age, driving experience and depressive symptoms when $20 \leqq P H Q-9 \leqq 27$ using SPSS.

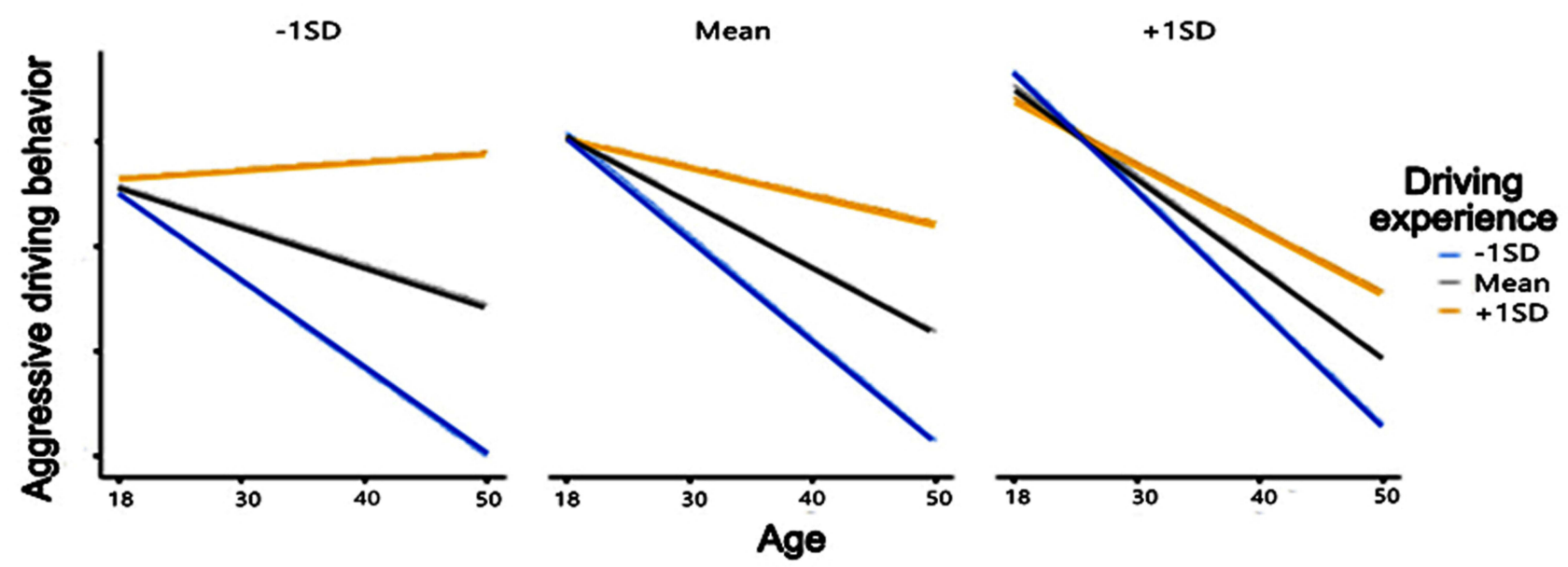

Figure 7 The simple effect test of age, driving experience and neuroticism using Jamovi software.

traits could be undertaken using a questionnaire such as the Eysenck personality Questionnaire, ${ }^{131}$ or Five-Factory Inventory. ${ }^{132}$ Additionally, a prevention logo or tips to warn about aggressive driving could be sent to targeted drivers using digital messages, such as Wei-chat messages in China. Such strategies have proven effective in health promotion strategies, ${ }^{133-136}$ enabling any potentially aggressive driving individuals to prevent activating their aggressive tendencies due to early awareness strategies. 


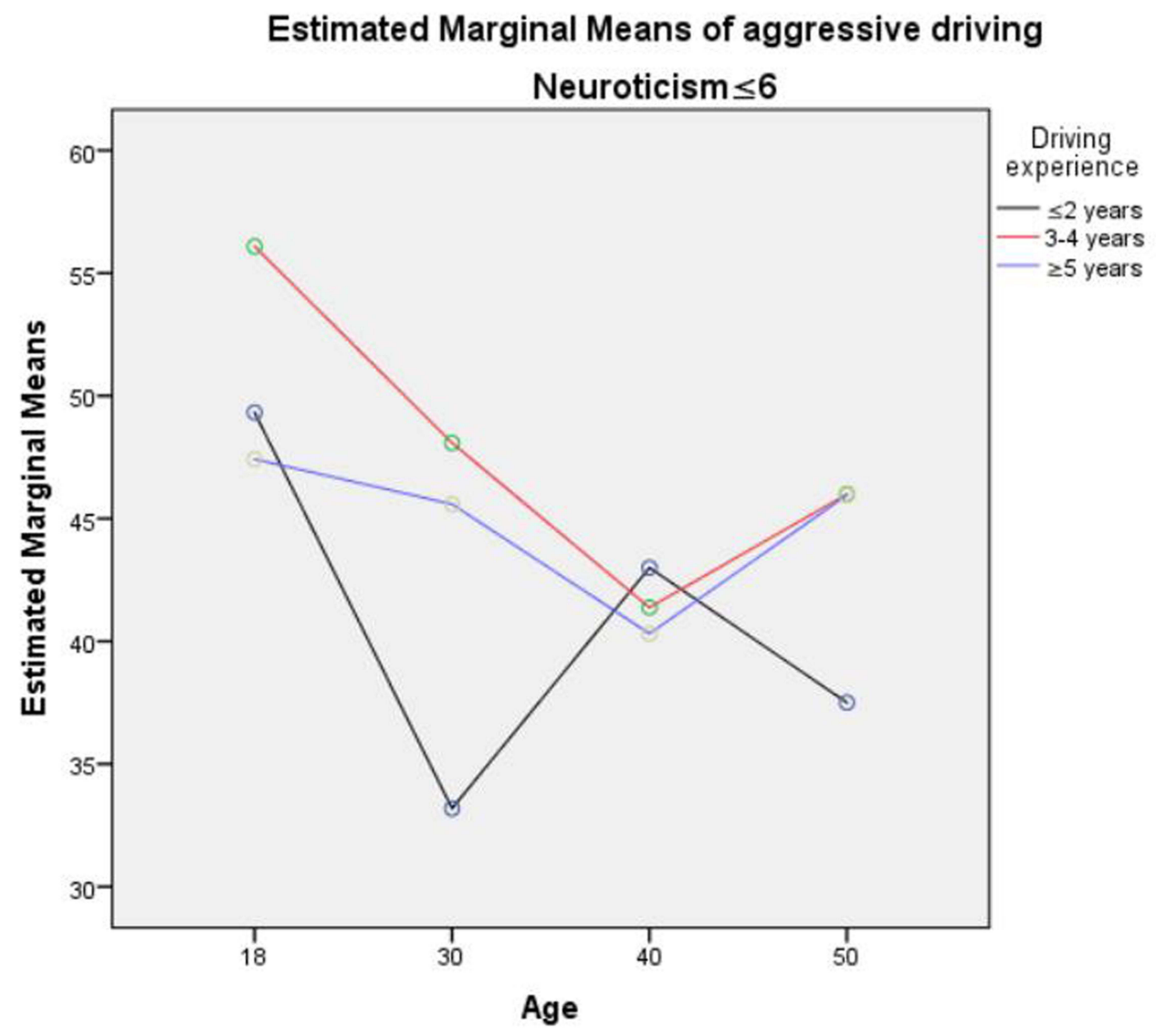

Figure 8 The simple effect of age, driving experience and depression symptoms when neuroticism $\leqq 6$ using SPSS.

\section{Limitations}

Despite the notable contributions of the current study to aggressive driving research, there are also real challenges in understanding the role of aggressive human factors in the real traffic environment. First, the cross-sectional design did not allow for the deduction of cause and effect, thus longitudinal research would be required. Second, the measurement of aggressive driving was derived from selfreporting measures, and more objective assessment methods could be used in future. Investigations. Third, external validation of the results required the testing of additional samples. Furthermore, the driving experience was measured using the number of driving years, whereas annual mileage should be considered in future studies. Finally, the study did not fully control the influencing factors of aggressive driving in the real world. There could be more confounding factors for road traffic accidents due to aggressive driving behavior in the complex dynamic traffic environment, such as the drivers' cognitive confounders (for example, attention, working memory and decision making processes) were not feasible to be included in a retrospective investigation. Therefore, the external validity of this study needs a great deal more consideration.

\section{Conclusion}

It is crucial to identify which demographic drivers more prone to contribute to accident-related conditions, as these findings can inform risky driver prevention and treatment to enhance traffic safety. First, this study suggested that age influenced aggressive driving behaviors negatively. However, veteran drivers (elderly and those with more extensive driving experience) could exhibit more risky driving behaviors than younger and inexperienced drivers when experiencing depressive symptoms. The findings allow for a greater understanding of the relationship between age and driving safety and indicated that elderly and experienced drivers require much more care and consideration for their mental health conditions to ensure traffic safety.

Further, the results confirmed that neuroticism influenced aggression in the context of the driving environment. Higher neuroticism drivers should be considered 


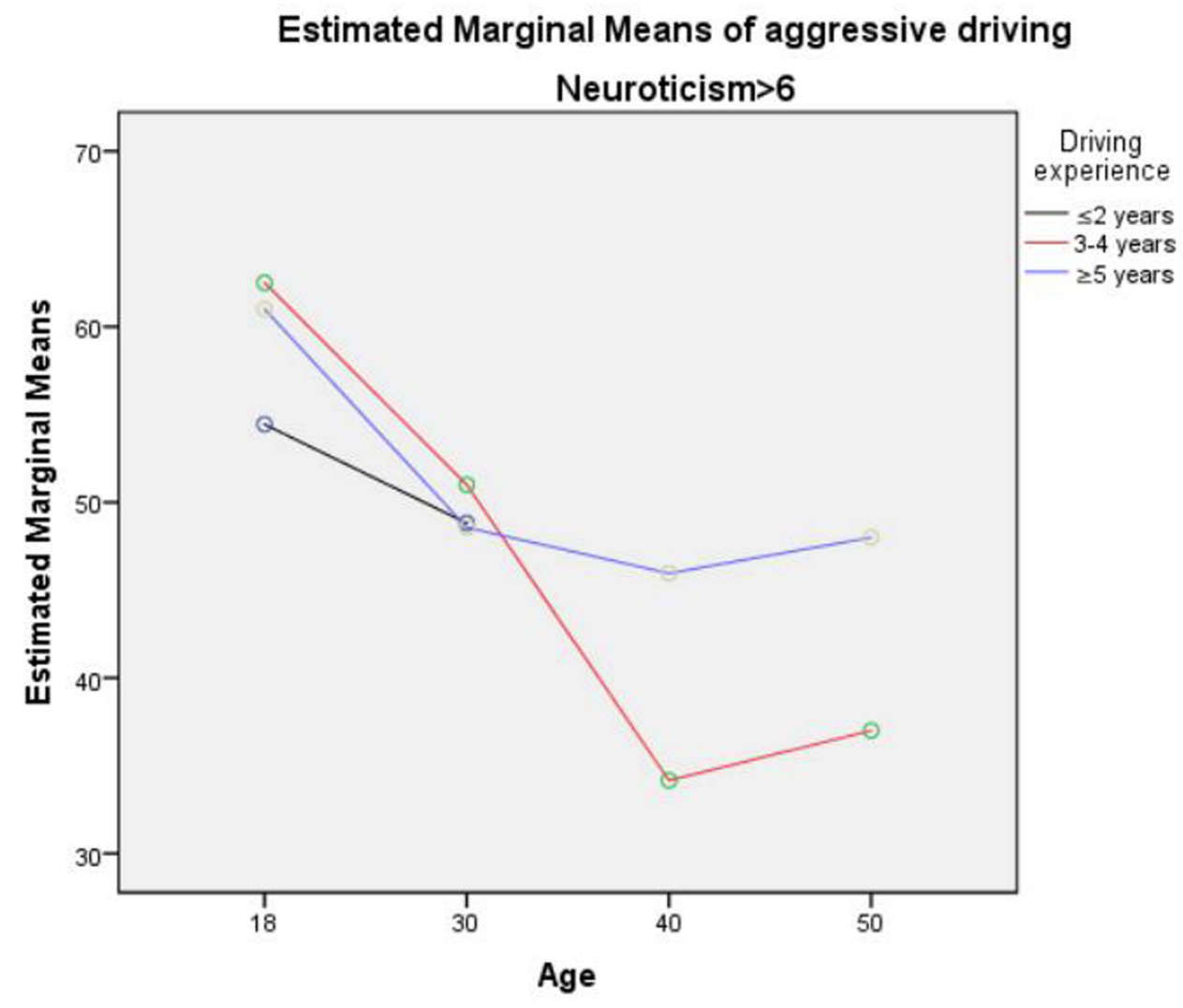

Non-estimable means are not plotted

Figure 9 The simple effect of age, driving experience and neuroticism when neuroticism $>6$ using SPSS.

worthwhile targets for preventing aggressive driving and ensuring roadside safety. Additionally, drivers with more vast driving experience displaying neuroticism were much more aggressive than less experienced drivers experiencing neuroticism. In particular, safety program should be directed at experienced drivers with higher neuroticism trait.

Psychological treatments can be considered a traffic safety strategy, together with vehicle operation regulations and traffic rules education. Contrary to our common sense, veteran drivers could be riskier when experiencing depression symptoms or neuroticism. To our knowledge, this is the first study reporting that veteran drivers were more risky drivers when experiencing particular psychological states. The findings suggested that mental health conditions and personality traits should be considered when traffic safety programs are developed and applied. Mobile digital interventions could be sent to the potentially risky drivers, which would be safe and broadly feasible as a tool to prevent aggressive driving behavior in the background of COVID-19.

\section{Disclosure}

The authors report no conflicts of interest in this work.

\section{References}

1. Haghpanahan H, Lewsey J, Mackay DF, et al. An evaluation of the effects of lowering blood alcohol concentration limits for drivers on the rates of road traffic accidents and alcohol consumption: a natural experiment. Lancet. 2019;393(10169):321-329. doi:10.1016/S01406736(18)32850-2

2. Murray CJL, Vos T, Lozano R. Disability-adjusted life years (dalys) for 291 diseases and injuries in 21 regions, 1990-2010: a systematic analysis for the global burden of disease study 2010. Lancet. 2012;380:2197-2223. doi:10.1016/S0140-6736(12)61689-4

3. Gabbe BJ, Lyons RA, Fitzgerald MC, Judson R, Richardson J, Cameron PA. Reduced population burden of road transport-related major trauma after introduction of an inclusive trauma system. Ann Surg. 2015;261(3):565-572. doi:10.1097/SLA.0000000000000522

4. Ebel BE, Mack C, Diehr P, Rivara FP. Lost working days, productivity, and restraint use among occupants of motor vehicles that crashed in the United States. Inj Prev. 2004;10(5):314-319. doi:10.1136/ ip.2004.005850

5. Bastida JL, Aguilar PS, Gonzalez BD. The economic costs of traffic accidents in Spain. J Trauma. 2004;56(4):883-888; discussion 888889. doi:10.1097/01.TA.0000069207.43004.A5

6. World Health Organization. Road traffic injuries. Available from: https://www.who.int/news/item/05-05-2019-advocates-around-theworld-speakup-for-roadsafety. Accessed December 10, 2021. 
7. Chu W, Wu C, Atombo C, Zhang H, Ozkan T. Traffic climate, driver behaviour, and accidents involvement in China. Accid Anal Prev. 2019;122:119-126. doi:10.1016/j.aap.2018.09.007

8. Huang H, Chang F, Schwebel DC, et al. Improve traffic death statistics in China. Science. 2018;362(6415):650. doi:10.1126/ science.aav5117

9. Wang L, Ning P, Yin P, et al. Road traffic mortality in China: analysis of national surveillance data from 2006 to 2016. Lancet Public Health. 2019;4:e245-e255. doi:10.1016/S2468-2667(19)30057-X

10. Podder V, Morita T, Tanimoto T. Reducing road traffic accidents in Bangladesh. Lancet. 2019;393(10169):315. doi:10.1016/ S0140-6736(18)32758-2

11. Zinzow HM, Jeffirs SM. Driving aggression and anxiety: intersections, assessment, and interventions. J Clin Psychol. 2018;74 (1):43-82. doi:10.1002/jclp.22494

12. Safety AFfT. Aggressive Driving: Research Update. Washington, DC: AAA Foundation for Traffic Safety; 2009.

13. Özkan T, Lajunen T, Parker D, Sümer N, Summala H. Symmetric relationship between self and others in aggressive driving across gender and countries. Traffic Inj Prev. 2010;11(3):228-239. doi: $10.1080 / 15389581003788864$

14. Paleti R, Eluru N, Bhat CR. Examining the influence of aggressive driving behavior on driver injury severity in traffic crashes. Accid Anal Prev. 2010;42(6):1839-1854. doi:10.1016/j.aap.20 10.05.005

15. Todd KH. Commentary: strategies to combat aggressive driving. Ann Emerg Med. 1999;34(6):800-801. doi:10.1016/S0196-064 4(99)70111-3

16. Ma Y, Gu X, Yu Y, Khattakc AJ, Chen S, Tang K. Identification of contributing factors for driver's perceptual bias of aggressive driving in China. Sustainability. 2021;13(2):766. doi:10.3390/ su13020766

17. Ma C, Hao W, Xiang W, Yan W. The impact of aggressive driving behavior on driver-injury severity at highway-rail grade crossings accidents. $J$ Adv Transp. 2018;2018:1-10.

18. Shinar D. Aggressive driving: the contribution of the drivers and the situation. Transp Res Part F Traffic Psychol Behav. 1998;1:137-160. doi:10.1016/S1369-8478(99)00002-9

19. Fakhry SM, Salaita K. Aggressive driving: a preliminary analysis of a serious threat to motorists in a large metropolitan area. J Trauma Inj Infect Crit Care. 2002;52:217-224. doi:10.1097/ 00005373-200202000-00003

20. Human aggression, 2nd edition [press release]. New York: Plenum Press; 1994.

21. Zahid M, Chen Y, Khan S, Jamal A, Ijaz M, Ahmed T. Predicting risky and aggressive driving behavior among taxi drivers: do spatio-temporal attributes matter? Int $J$ Environ Res Public Health. 2020;17(11):3937. doi:10.3390/ijerph17113937

22. Alver Y, Demirel MC, Mutlu MM. Interaction between socio-demographic characteristics: traffic rule violations and traffic crash history for young drivers. Accid Anal Prev. 2014;72:95-104. doi:10.1016/j.aap.2014.06.015

23. Shi J, Tao L, Li X, Xiao Y, Atchley P. A survey of taxi drivers aberrant driving behavior in Beijing. J Transp Saf Secur. 2014;6:34-43.

24. Lawton R, Parker D, Manstead ASR, Stradling SG. The role of affect in predicting social behaviours: the case of road traffic violations. J Appl Soc Psychol. 1997;27:1258-1276. doi:10.1111/ j.1559-1816.1997.tb01805.x

25. Elander J, West R, French D. Behavioral correlates of individual differences in road-traffic crash risk: an examination method and findings. Psychol Bull. 1993;113(2):279-294. doi:10.1037/00332909.113.2.279

26. Perepjolkina V, Reñge V. Drivers' age, gender, driving experience and aggressiveness as predictors of aggressive driving behaviour Signum Tempor. 2011;4:62-72.
27. Wickens CM, Mann RE, Stoduto G, Ialomiteanu A, Smart RG. Age group differences in self-reported aggressive driving perpetration and victimization. Transp Res Part F Traffic Psychol Behav. 2011;14(5):400-412. doi:10.1016/j. trf.2011.04.007

28. Zhang H, Qu W, Ge Y, Sun X, Zhang K. Effect of personality traits, age and sex on aggressive driving: psychometric adaptation of the driver aggression indicators scale in China. Accid Anal Prev. 2017;103:29-36. doi:10.1016/j.aap.2017.0 3.016

29. Guo M, Wei W, Liao G, Chu F. The impact of personality on driving safety among Chinese high-speed railway drivers. Accid Anal Prev. 2016;92:9-14. doi:10.1016/j.aap.2016.03.014

30. Archer J. Does sexual selection explain human sex differences in aggression? Behav Brain Sci. 2009;32(3-4):249-266; discussion 266-311. doi:10.1017/S0140525X09990951

31. Ben Khelil M, Belghith M, Chraiti A, Gharbaoui M, Laadhari N, Hamdoun M. Workplace homicides in northern Tunisia: a 15-year study (2003-2017). Am J Forensic Med Pathol. 2019;40 (4):366-370. doi:10.1097/PAF.0000000000000498

32. Sousa Santana V, Dias EC, Oliveira GL, Moura MCP, Nobre LCDC, Machado JMH. Accidentes de trabajo fatales violencia interpersonal en brasil, 2000-2010. Salud Colect. 2013;9:139-149. doi:10.18294/sc.2013.28

33. Jenkins EL. Homicide against women in the workplace. $J \mathrm{Am}$ Med Womens Assoc. 1996;51:118-119.

34. Darwin C. The descent of man, and selection in relation to sex; 1871/1901. Available from: http://www.infidels.org/library/histor ical/charles. Accessed December 2, 2021

35. Eagly AH, Steffen VJ. Gender stereotypes stem from the distribution of women and men into social roles. J Pers Soc Psychol. 1984;46:735-754. doi:10.1037/0022-3514.46.4.735

36. Wood W, Eagly AH. A cross-cultural analysis of the behavior of women and men: implications for the origins of sex differences. Psychol Bull. 2002;128(5):699-727. doi:10.1037/0033-2909.128. 5.699

37. Staniloiu A, Markowitsch H. Gender differences in violence and aggression - a neurobiological perspective. Procedia Soc Behav Sci. 2012;33:1032-1036. doi:10.1016/j.sbspro.2012.01.279

38. Braly AM, Parent MC, DeLucia PR. Do threats to masculinity result in more aggressive driving behavior? Psychol Men Masc. 2018;19(4):540-546. doi:10.1037/men0000135

39. Hennessy DA, Wiesenthal DL. Aggression, violence, and vengeance among male and female drivers. Transp Q. 2002;56(4):65-75.

40. Jia Y, Zhang L, Duan Y, Zhang J, Cui X, Li L. Associations of anger while driving and driving style with aggressive behaviors in drivers. Chin J Public Health. 2016;32(10):1373-1377.

41. Feng Z, Yuan H, Liu J, Zhang W, Liu H. Influence of driver personal characteristics on vehicle velocity. J Traffic Transp Eng. 2012;12(6):90-96.

42. Gwyther H, Holland C. The effect of age, gender and attitudes on self-regulation in driving. Accid Anal Prev. 2012;45:19-28. doi:10.1016/j.aap.2011.11.022

43. Lajunen T, Parker D. Are aggressive people aggressive drivers? A study of the relationship between self-reported general aggressiveness, driver anger, and aggressive driving. Accid Anal Prev. 2001;33:243-255. doi:10.1016/S0001-4575(00)00039-7

44. Li F, Li C, Long Y, Zhan C, Hu B. The analysis of psychosocial factors of drivers' aggressive driving behavior. J Sichuan Univ. 2004;35(4):568-570.

45. Iancu AE, Hogea A, Olteanu AF. The association between personality and aggressive driving_ a meta-analysis. Rom $\mathrm{J} \mathrm{Appl}$ Psychol. 2016;18:24-32.

46. Totkova Z. Interconnection between driving style, traffic locus of control, and impulsivity in Bulgarian drivers. Behav Sci (Basel). 2020;10(2):58. 
47. McCutcheon VV, Agrawal A, Heath AC, et al. Functioning of alcohol use disorder criteria among men and women with arrests for driving under the influence of alcohol. Alcohol Clin Exp Res. 2011;35(11):1985-1993. doi:10.1111/j.15300277.2011.01550.x

48. McCutcheon VV, Heath AC, Edenberg HJ, et al. Alcohol criteria endorsement and psychiatric and drug use disorders among dui offenders: greater severity among women and multiple offenders. Addict Behav. 2009;34(5):432-439. doi:10.1016/j.addbeh.2008. 12.003

49. Agbaria Q, Natur N. The relationship between violence in the family and adolescents aggression: the mediator role of self-control, social support, religiosity, and well-being. Child Youth Serv Rev. 2018;91:447-456. doi:10.1016/j.childyouth.2018.06.016

50. Xie D, Xie Z. Adolescents' online anger and aggressive behavior: moderating effect of seeking social support. Soc Behav Pers. 2019;47(6):1-9. doi:10.2224/sbp.7976

51. Otte S, Lang FU, Vasic N, et al. Are depressed people aggressive people? Differences between general population and depressive patients. Psychother Psychosom Med Psychol. 2017;67(1):19-25. doi:10.1055/s-0042-120411

52. Meyrueix L, Durham G, Miller J, Smalley KB, Warren JC. Association between depression and aggression in rural women. $J$ Health Dispar Res Pract. 2015;8(4):136-144.

53. Krizan Z, Herlache AD. Sleep disruption and aggression: implications for violence and its prevention. Psychol Violence. 2016;6 (4):542-552. doi:10.1037/vio0000018

54. Van Veen MM, Rutters F, Spreen M, Lancel M. Poor sleep quality at baseline is associated with increased aggression over one year in forensic psychiatric patients. Sleep Med. 2020;67:1-6. doi:10.1016/j.sleep.2019.11.1183

55. Bureau Tmopstm. Road Traffic Accident Statistics Report; 2014.

56. Daily TPs. GDP figures in the year of 2018 in 31 provinces in China; 2019. Available from: https://baijiahao.baidu.com $/ \mathrm{s}$ ?id= $1626820447641536330 \& w f r=$ spider \&for $=$ pc . Accessed December $10,2021$.

57. Website or. Statistics of civil automobile ownership in China from 2010 to 2019 (with provincial data); 2021. Available from: http:// data.chinabaogao.com/qiche/2021/0215312942021.html. Accessed December 2, 2021.

58. Statistics JPBo. Statistical bulletin of economic and social development of Jiangsu province in 2018; 2019. Available from: http:// www.js.gov.cn/art/2019/3/25/art_64797_8284235.html. Accessed December 2, 2021.

59. The rank of cities' GDP in Jiangsu province in 2018. Available from: https://baijiahao.baidu.com/s? id = $1623891449381212206 \& \mathrm{wfr}=$ spider $\&$ for $=$ pc\&searchword $=$ $2018 \%$ E6\%B 1\%9F\%E $8 \% 8$ B $\% 8$ F $\%$ E7\% 9 C $\% 81 \%$ E $5 \% 86 \%$ $85 \mathrm{gdp} \% \mathrm{E} 6 \% 8 \mathrm{E} \% 92 \% \mathrm{E} 5 \% 90 \% 8 \mathrm{D} \& \mathrm{qq}-\mathrm{pf}$-to=pcqq.c2c. Accessed January 28, 2019.

60. Website S. Jiangsu's GDP for 2018; 2019. Available from: https:// www.sohu.com/a/295786184_120046606. Accessed December 2, 2021.

61. qq.com. GDP ranking of districts in Wuxi area. Available from: https://new.qq.com/rain/a/20200520A0ET7T00. Accessed December $10,2021$.

62. Government China, 2016. [Command from the Ministry of public security of the people's Republic of China]. Available from: http://www.gov.cn/gongbao/content/2016/content_5070752.htm. Accessed December 2, 2021.

63. Bureau CsT. No.74 noticement of taxation on luxury car; 2016.

64. Luo Y. Analysis and Research of Aggressive Driving Behaviour at the Road Traffic. Planning and Management of Traffic\&Transportation, Southwest Jiaotong University; 2009. Available from: http://cdmd.cnki.com.cn/Article/CDMD-106132009217466.htm. Accessed December 17, 2021.
65. Williams N. The gad-7 questionnaire. Occup Med (Chic Ill). 2014;64(3):224. doi:10.1093/occmed/kqt161

66. Spitzer RL, Kroenke K, Williams JBW, Löwe B. A brief measure for assessing generalized anxiety disorder: the gad-7. Arch Intern Med. 2006;166(10):1092-1097. doi:10.1001/archinte.166.10.1092

67. Patrick S, Connick P. Psychometric properties of the phq-9 depression scale in people with multiple sclerosis: a systematic review. PLoS One. 2019;14(2):e0197943.

68. Wang W, Bian Q, Zhao Y. Reliability and validity of the Chinese version of the patient health questionnaire (phq-9) in the general population. Gen Hosp Psychiatry. 2014;36(5):539-544. doi:10. 1016/j.genhosppsych.2014.05.021

69. Eysenck HJ, Eysenck SBG. Manual of the Eysenck Personality Questionnaire. London: Hodder and Stoughton; 1975.

70. Eysenck SBG, Eysenck HJ, Barrett P. A revised version of the psychoticism scale. Pers Individ Dif. 1985;6(1):21-29. doi:10. 1016/0191-8869(85)90026-1

71. Backhaus J, Junghanns K, Broocksa A, Riemann D, Hohagen F. Test-retest reliability and validity of the Pittsburgh sleep quality index in primary insomnia. J Psychosom Res. 2002;53:737-740. doi:10.1016/S0022-3999(02)00330-6

72. Tsai PS, Wang SY, Wang MY, et al. Psychometric evaluation of the Chinese version of the Pittsburgh sleep quality index (CPSQI) in primary insomnia and control subjects. Qual Life Res. 2005;14 (8):1943-1952. doi:10.1007/s11136-005-4346-x

73. Bohn M, Babor T, Kranzler H. The alcohol use disorders identification test (audit): validation of a screening instrument for use in medical settings. J Stud Alcohol. 1995;56(4):423-432. doi:10.15 288/jsa.1995.56.423

74. Zhang C, Yang G, Li Z. Reliability and validity of the Chinese version on alcohol use disorders identification test. Chin J Epidemiol. 2017;38:1064-1067.

75. Slevin ML, Nichols SE, Downer SM, et al. Emotional support for cancer patients: what do patients really want? $\mathrm{Br} J$ Cancer. 1996;74:1275-1279. doi:10.1038/bjc.1996.529

76. Wills TA, Margaret C. Social support and interpersonal relationships. Prosoc Behav Rev Pers Soc Psychol. 1991;12: 265-289.

77. Krause N. Social support, stress, and well-being among older adults. J Gerontol. 1986;41(4):512-519. doi:10.1093/geronj/41.4.512

78. House JS. Work stress and social support. Reading. AddisonWesley; 1981.

79. Heaney CA, Israel BA. Social networks and social support. In: Glanz K, Rimer BK, Viswanath K, editors. Health Behavior and Health Education: Theory, Research, and Practice (4th Ed.). San Francisco, CA: Jossey-Bass; 2008.

80. Xiao S-Y. The theoretical basis and application research of social support rating scale. Chin J Clin Psychiatry. 1994;4:98-100.

81. Frisch R. Statistical confluence analysis by means of complete regression systems. Oslo University: Economic Institute; 1934.

82. Queen JP, Quinn GP, Keough MJ. Experimental Design and Data Analysis for Biologists. Cambridge: Cambridge University Press; 2002.

83. Administration NHS. Critical Reasons for Crashes Investigated in the National Motor Vehicle Crash Causation Survey; 2015.

84. Lewin I. Driver training: a perceptual-motor skill approach. Ergonomics. 1982;25:917-924. doi:10.1080/00140138208925051

85. West R, French D, Kemp R, Elander J. Direct observation of driving, self reports of driver behaviour and accident involvement. Ergonomics. 1993;36:557-567. doi:10.1080/00140139308967912

86. Lajunen T, Summala H. Can we trust self-reports of driving? Effects of impression management on driver behaviour questionnaire responses. Transp Res F. 2003;6:97-107. doi:10.1016/ S1369-8478(03)00008-1

87. Krahé B. Predictors of women's aggressive driving behavior. Aggress Behav. 2005;31(6):537-546. doi:10.1002/ab.20070 
88. Blain-Arcaro C, Vaillancourt T. Longitudinal associations between depression and aggression in children and adolescents. J Abnorm Child Psychol. 2017;45(5):959-970. doi:10.1007/ s10802-016-0204-2

89. Kim IJ, Ge X, Brody GH, Conger RD, Gibbons FX, Simons RL. Parenting behaviors and the occurrence and co-occurrence of depressive symptoms and conduct problems among African American children. J Fam Psychol. 2003;17(4):571-583. doi:10.1037/0893-3200.17.4.571

90. Snyder J, Prichard J, Schrepferman L, Patrick MR, Stoolmiller M. Child impulsiveness - inattention, early peer experiences, and the development of early onset conduct problems. J Abnorm Child Psychol. 2004;32(6):579-594. doi:10.1023/B:JACP.0000047208. 23845.64

91. Piko BF, Pinczés T. Impulsivity, depression and aggression among adolescents. Pers Individ Dif. 2014;69:33-37. doi:10. 1016/j.paid.2014.05.008

92. Moeller FG, Barratt ES, Dougherty DM, Schmitz JM, Swann AC. Psychiatric aspects of impulsivity. Am J Psychiatry. 2001;158 (11):1783-1793. doi:10.1176/appi.ajp.158.11.1783

93. Association AP. Diagnostic and Statistical Manual of Mental Disorders (DSM-5). American Psychiatric Pub; 2013.

94. Serretti A, De Ronchi D, Olgiati P. Irritable mood and subthreshold hypomanic episodes correlate with more severe major depression. Neuropsychobiology. 2021;80:425-435.

95. Liu Q, Cole DA. The association of phasic irritability (aggressive outbursts) and tonic irritability (irritable mood) to depression occurrences, symptoms, and subtypes. J Affect Disord. 2021;293:9-18. doi:10.1016/j.jad.2021.06.012

96. Leadbeater BJ, Homel J. Irritable and defiant sub-dimensions of odd: their stability and prediction of internalizing symptoms and conduct problems from adolescence to young adulthood. J Abnorm Child Psychol. 2015;43(3):407-421. doi:10.1007/ s10802-014-9908-3

97. Stringaris A, Goodman R. Longitudinal outcome of youth oppositionality: irritable, headstrong, and hurtful behaviors have distinctive predictions. $J$ Am Acad Child Adolesc Psychiatry. 2009;48(4):404-412. doi:10.1097/CHI.0b013e3181984f30

98. Lopez-Morinigo JD, Boldrini M, Ricca V, Oquendo MA, BacaGarcia E. Aggression, impulsivity and suicidal behavior in depressive disorders: a comparison study between New York city (US), Madrid (Spain) and Florence (Italy). J Clin Med. 2021;10(14):3057. doi:10.3390/jcm10143057

99. Dorfman HM, Meyer-Lindenberg A, Buckholtz JW. Neurobiological mechanisms for impulsive-aggression: the role of maoa. Curr Top Behav Neurosci. 2014;17:297-313.

100. Bozkurt M, Evren C, Yilmaz A, Can Y, Cetingok S. Aggression and impulsivity in different groups of alcohol and heroin dependent inpatient men. Bull Clin Psychopharmacol. 2016;23 (4):335-344. doi:10.5455/bcp.20130127021314

101. Van Praag H. Anxiety and increased aggression as pacemakers of depression. Acta Psychiatr Scand. 1998;98:81-88.

102. Kallioniemi H, Syvälahti E. Citalopram, a specific inhibitor of serotonin re-uptake in treatment of psychotic and borderline patients. Nord J Psychiatr. 1993;47:79-84. doi:10.3109/08 039489309102786

103. Fava M, Rosenbaum JF, Pava JA, McCarthy MK, Steingard RJ, Bouffides E. Anger attacks in unipolar depression. Part 1: clinical correlates and response to fluoxetine treatment. Am J Psychiatry. 1993;150:1158-1163. doi:10.1176/ajp.150.8.1158

104. Castrogiovanni P, Di Muro A, Maremanni I. Fluoxetine reduces aggressive behavior in depressive outpatients. New Trends Exp Clin Psychiat. 1992;8:57.

105. Morcos N, Maixner S, Maixner DF. Electroconvulsive therapy for bipolar depression in older adults. J ECT. 2021;37(3):182-188. doi:10.1097/YCT.0000000000000755
106. Morais A, Liu TT, Qin T, et al. Vagus nerve stimulation inhibits cortical spreading depression exclusively through central mechanisms. Pain. 2020;161(7):1661-1669. doi:10.1097/j.pain. 0000000000001856

107. Schlaepfer T, Kosela M. Novel physical treatments for major depression: vagus nerve stimulation, transcranial magnetic stimulation and magnetic seizure therapy. Curr Opin Psychiatry. 2004;17:15-20. doi:10.1097/00001504-2004010 00-00004

108. Fitzgerald PB, Sritharan A, Daskalakis ZJ, de Castella AR, Kulkarni J, Egan G. A functional magnetic resonance imaging study of the effects of low frequency right prefrontal transcranial magnetic stimulation in depression. J Clin Psychopharmacol. 2007;27(5):488-492. doi:10.1097/jcp.0b013e318151521c

109. Duncan C, Salvacion C, Richardson S. Transcranial magnetic stimulation and depression. Nurse Pract. 2021;46(2):13-15. doi:10.1097/01.NPR.0000724492.63143.cc

110. Jørgensen CK, Juul S, Siddiqui F, et al. Tricyclic antidepressants versus 'active placebo', placebo or no intervention for adults with major depressive disorder: a protocol for a systematic review with meta-analysis and trial sequential analysis. Syst Rev. 2021;10(1). doi:10.1186/s13643-021-01789-0

111. Quitkin F, Rifkin A, Klein DF. Monoamine oxidase inhibitors. A review of antidepressant effectiveness. Arch Gen Psychiatry. 1979;36(7):749-760. doi:10.1001/archpsyc.1979.01780070027 003

112. Thom RP, Alexander JL, Baron D, et al. Selective serotonin reuptake inhibitors: how long is long enough? J Psychiatr Pract. 2021;27 (5):361-371. doi:10.1097/PRA.0000000000000578

113. Gong L. Evaluation on the rational use of drugs for selective serotonin and norepinephrine reuptake inhibitors (ssnri). In: Evaluation and Analysis of Drug Use in Hospitals of China. Vol. 5; 2009:323-325.

114. Jia Y, Wang X, Cheng Y. Relaxation therapy for depression: an updated meta-analysis. J Nerv Ment Dis. 2020;208(4):319-328. doi:10.1097/NMD.0000000000001121

115. Karyotaki E, Efthimiou O, Miguel C. Internet-based therapy for depression found effective. JAMA Psychiatry. 2021;78 (4):361-371. doi:10.1001/jamapsychiatry.2020.4364

116. Carpenter KM, Stoner SA, Mundt JM, Stoelb B. An online self-help cbt intervention for chronic lower back pain. Clin $J$ Pain. 2012;28:14-22. doi:10.1097/AJP.0b013e31822363db

117. Atema V, van Leeuwen M, Oldenburg HSA, van Beurden M, Hunter MS, Aaronson NK. An internet-based cognitive behavioral therapy for treatment-induced menopausal symptoms in breast cancer survivors: results of a pilot study. Menopause. 2017;24(7):762-767. doi:10.1097/GME.0000000000000836

118. Miki A, Lau MA, Moradian H. An open trial of the effectiveness, program usage, and user experience of internet-based cognitive behavioural therapy for mixed anxiety and depression for healthcare workers on disability leave. J Occup Environ Med. 2021;63 (10):865-874. doi:10.1097/JOM.0000000000002248

119. Rusting CL. Personality, mood, and cognitive processing of emotional information: three conceptual frameworks. Psychol Bull. 1998;124(2):165. doi:10.1037/0033-2909.124.2.165

120. Arthur W, Doverspike D. Predicting motor vehicle crash involvement from a personality measure and a driving knowledge test. J Prev Interv Community. 2001;22(1):35-42. doi:10.1080/ 10852350109511209

121. Jovanović D, Lipovac K, Stanojević P, Stanojević D. The effects of personality traits on driving-related anger and aggressive behaviour in traffic among Serbian drivers. Transp Res Part F Traffic Psychol Behav. 2011;14(1):43-53. doi:10.1016/j.trf.2010.09.005

122. Jones SE, Miller JD, Lynam DR. Personality, antisocial behavior, and aggression: a meta-analytic review. J Crim Justice. 2011;39 (4):329-337. doi:10.1016/j.jcrimjus.2011.03.004 
123. Ball L, Tully R, Egan V. The influence of impulsivity and the dark triad on self-reported aggressive driving behaviours. Accid Anal Prev. 2018;120:130-138. doi:10.1016/j.aap.2018.08.010

124. Shen B, Ge Y, Qu W, Sun X, Zhang K. The different effects of personality on prosocial and aggressive driving behaviour in a Chinese sample. Res Part F Traffic Psychol Behav. 2018;56:268-279. doi:10.1016/j.trf.2018.04.019

125. Smith P, Waterman M, Ward N. Driving aggression in forensic and non-forensic populations: relationships to self-reported levels of aggression, anger and impulsivity. Br J Psychol. 2006;97(Pt 3):387-403. doi:10.1348/000712605X79111

126. Barratt ES. Violence and Mental Disorders: Developments in Risk Assessment. Chicago: University of Chicago Press; 1994.

127. Krakowski M, Czobor P, Carpenter MD, et al. Community violence and inpatient assaults: neurobiological deficits. J Neuropsychiatry Clin Neurosci. 1997;9:549-555. doi:10.1176/jnp.9.4.549

128. Blair RJR, Jones L, Clark F, Smith M. The psychopathic individual: alack of responsiveness to distress cues? Psychophysiology. 1997;34:192-198. doi:10.1111/j.1469-8986.1997.tb02131.x

129. Blair RJR. A cognitive developmental approach to morality: investigating the psychopath. Cognition. 1995;57:1-29. doi:10. 1016/0010-0277(95)00676-P

130. Blair RJR, Morton J. Putting cognition into sociopathy. Brain Behav Sci. 1995;18:548.

131. Nasreldin M, Gohar SM, Abdel Samie M, Madbouly N. Psychiatric morbidity and personality characteristics of detained adolescents with delinquent behavior: a cross-sectional analytical study. J Nerv Ment Dis. 2020;208(12):989-996. doi:10.1097/ NMD.0000000000001244
132. Janssens H, De Zutter P, Geens T, Vogt G, Braeckman L. Do personality traits determine work engagement? Results from a Belgian study. J Occup Environ Med. 2019;61(1):29-34. doi:10.1097/JOM.0000000000001458

133. Fritsch CG, Ferreira PH, Prior JL, McLachlan AJ, Ferreira ML. Effects of using text message interventions for the management of musculoskeletal pain: a systematic review. Pain. 2020;161 (11):2462-2475. doi:10.1097/j.pain.0000000000001958

134. Palermo TM, de la Vega R, Murray C, Law E, Zhou C. A digital health psychological intervention (webmap mobile) for children and adolescents with chronic pain: results of a hybrid effectiveness-implementation stepped-wedge cluster randomized trial. Pain. 2020;161(12):2763-2774. doi:10.1097/j.pain.0000 000000001994

135. Stahl I, Katsman A, Zaidman M, Keshet D, Sigal A, Eidelman M. Reliability of smartphone-based instant messaging application for diagnosis, classification, and decision-making in pediatric orthopedic trauma. Pediatr Emerg Care. 2017:1-4. doi:10.1097/PEC. 000000000000716

136. Kim S, Lee S. Effects of government-driven smartphone text messages on public compliance with covid-19 preventative measures. Comput Inform Nurs. 2021;39(10):527-537.
Psychology Research and Behavior Management

\section{Publish your work in this journal}

Psychology Research and Behavior Management is an international, peer-reviewed, open access journal focusing on the science of psychology and its application in behavior management to develop improved outcomes in the clinical, educational, sports and business arenas. Specific topics covered in the journal include: Neuroscience, memory and decision making; Behavior modification and management; Clinical

\section{Dovepress}

applications; Business and sports performance management; Social and developmental studies; Animal studies. The manuscript management system is completely online and includes a very quick and fair peer-review system, which is all easy to use. Visit http://www. dovepress.com/testimonials.php to read real quotes from published authors. 